\title{
Macrolides rapidly inhibit red blood cell invasion by the human malaria parasite, Plasmodium falciparum
}

\author{
Danny W Wilson ${ }^{1,2,3,4^{*}}$, Christopher D Goodman ${ }^{5}$, Brad E Sleebs ${ }^{2,3}$, Greta E Weiss ${ }^{4}$, Nienke WM de Jong ${ }^{4}$, \\ Fiona Angrisano ${ }^{2,3,6}$, Christine Langer ${ }^{4}$, Jake Baum ${ }^{2,3,6}$, Brendan S Crabb ${ }^{3,4,7}$, Paul R Gilson ${ }^{4,7}$, \\ Geoffrey I McFadden ${ }^{5}$ and James G Beeson ${ }^{2,4,8^{*}}$
}

\begin{abstract}
Background: Malaria invasion of red blood cells involves multiple parasite-specific targets that are easily accessible to inhibitory compounds, making it an attractive target for antimalarial development. However, no current antimalarial agents act against host cell invasion.

Results: Here, we demonstrate that the clinically used macrolide antibiotic azithromycin, which is known to kill human malaria asexual blood-stage parasites by blocking protein synthesis in their apicoplast, is also a rapid inhibitor of red blood cell invasion in human (Plasmodium falciparum) and rodent (P. berghei) malarias. Multiple lines of evidence demonstrate that the action of azithromycin in inhibiting parasite invasion of red blood cells is independent of its inhibition of protein synthesis in the parasite apicoplast, opening up a new strategy to develop a single drug with multiple parasite targets. We identified derivatives of azithromycin and erythromycin that are better invasion inhibitors than parent compounds, offering promise for development of this novel antimalarial strategy.
\end{abstract}

Conclusions: Safe and effective macrolide antibiotics with dual modalities could be developed to combat malaria and reduce the parasite's options for resistance.

Keywords: Plasmodium, Merozoite, Invasion, Macrolide, Dual modality

\section{Background}

Malaria is a mosquito-borne parasitic disease, which causes nearly 600,000 deaths every year, mainly in children under 5 years of age in sub-Saharan Africa [1]. Most deaths are attributed to the most virulent human malaria parasite, Plasmodium falciparum. Current disease control measures rely on reducing exposure to the mosquito vector or treatment of clinical malaria cases with antimalarial drugs that target the disease causing blood-stage trophozoite; most of these act against the parasite's food vacuole or interfere with pyrimidine synthesis of maturing trophozoites [2-6]. Of major concern

\footnotetext{
* Correspondence: danny.wilson@adelaide.edu.au; beeson@burnet.edu.au ${ }^{1}$ Research Centre for Infectious Diseases, School of Biological Sciences, The University of Adelaide, Adelaide, South Australia

${ }^{2}$ Walter and Eliza Hall Institute of Medical Research, Parkville, Victoria 3050, Australia

Full list of author information is available at the end of the article
}

is the emerging development of drug resistance to the highly effective artemisinin family of compounds in Southeast Asia [7-9]. The prospect of widespread resistance to the most effective antimalarials becoming entrenched has led to the implementation of an emergency response plan by the World Health Organization (WHO) to prevent the spread of resistance and the inevitable increase in childhood mortality that would result [10]. This developing emergency highlights the urgent need for new antimalarials with novel mechanisms of action for use in combination with artemisinins, or to replace these drugs in the future.

Invasion of blood-stage merozoites into host cells has been proposed as a potential target for antimalarial chemotherapy [11-14]. Invasion of the extracellular merozoite into a new host erythrocyte (red blood cell) is a complex process requiring the coordinated interaction of unique parasite ligands, signaling pathways, and a 
form of gliding motility powered by an actomyosin motor. The multiple accessible targets make this essential stage of the lifecycle an attractive drug target. In addition, inhibition of merozoite invasion into the erythrocyte would lead to instant disruption of the parasite lifecycle and prevent sequestration, dormancy and commitment to the mosquito-transmissible gametocyte stage, which have been reported to occur after drug treatment with current first-line antimalarials [15-17]. Application of antimalarial drugs that target invasion has the potential to result in quicker resolution of clinical disease if used in combination with antimalarials that act at other developmental stages.

Few compounds have been directly tested for ability to inhibit $P$. falciparum merozoite invasion, partly through lack of tractable invasion inhibition assays. The ability to assess the invasion inhibitory potential of compounds and their biological activity in vitro has been greatly enhanced with the development of a robust and efficient merozoite purification protocol and techniques to directly measure invasion inhibition [13, 18-20], allowing the accurate and rapid assessment of a compounds invasion inhibitory potential. While some invasion inhibitory compounds (e.g. protease inhibitors [18, 21, 22], heparin [23] and calcium signaling modulators [23, 24]) have been reported, to date no drug used clinically to treat malaria has been shown to have inhibitory activity against merozoite invasion [13]. In this study, we have identified clinically relevant macrolide antibiotics and related compounds, exemplified by azithromycin, as rapid inhibitors of $P$. falciparum and $P$. berghei merozoite invasion in vitro. This represents the first time that a drug or drug class that has clinical applications against malaria has been demonstrated to inhibit parasite invasion of the red blood cell.

Antibiotics such as azithromycin and clindamycin (lincosamide antibiotic) have been proposed as potential partner drugs for artemisinin combination therapies owing to their extremely long half-life and good safety profile in vivo [25-29]. Both azithromycin and clindamycin inhibit apicoplast ribosomal protein synthesis of asexual blood-stage parasites at nanomolar (clinical) concentrations by binding to the apicoplast ribosomal $50 \mathrm{~S}$ subunit and blocking protein exit from the ribosome [30-32]. In vitro experiments at clinically relevant concentrations show that these antibiotics have a 'delayed death' drug response. Treated parasites grow normally during the first lifecycle under treatment. Only during the second post-treatment cycle (after replication and reinvasion) are drug effects observed. Parasite death at this stage is thought to result from the inheritance of a defective apicoplast that is unable to synthesize isoprenoid precursors required for development [31-33]. This 'delayed death' response currently limits the usefulness of azithromycin and clindamycin for the treatment of clinical disease as stand-alone drugs, and successful combinations with other antimalarials remain elusive [34].

In this study, we aimed to identify existing, and novel, compounds with invasion inhibitory activity that have the potential for development as effective antimalarials. We demonstrate that the antibiotic azithromycin can specifically inhibit merozoite invasion. Related macrolide antibiotics were also found to inhibit invasion, and the $\mathrm{IC}_{50}$ of invasion inhibitory activity could be lowered through modification. These results provide the basis for a novel strategy of antimalarial drug development by advancing compounds that have dual mechanisms of action. Given the established safety and low cost of macrolides in widespread clinical use, development of macrolides as antimalarials with dual modalities against merozoite invasion and protein translation in the apicoplast is a promising strategy to counter the rapid emergence of drug resistance.

\section{Results}

Azithromycin inhibits merozoite invasion

Application of the merozoite purification method of Boyle et al. [18] identified the macrolide antibiotic azithromycin as a candidate inhibitor of $P$. falciparum asexual blood-stage merozoite invasion of the host erythrocyte (see Fig. 1a for representation of assay setup, Fig. 2 for structure of azithromycin and other drugs used in this study). Initial screens indicated that the invasion inhibitory $\mathrm{IC}_{50}$ differed between azithromycin prepared in ethanol $(10 \mu \mathrm{M})$ or DMSO $(38 \mu \mathrm{M})$, suggesting that choice of vehicle can impact azithromycin potency in vitro. Comparative screens of azithromycin potency over different treatment times demonstrated that the $\mathrm{IC}_{50}$ of merozoite invasion assays (1 hour exposure to drug) were surprisingly similar to the in cycle ( 40 hour incubation, no invasion step, Fig. 1b) and 1 cycle (90 hour, one invasion step, Fig. 1c) assays $\left(\mathrm{IC}_{50}-\right.$ (drug prepared in ethanol) invasion $10 \mu \mathrm{m}$; in cycle $6 \mu \mathrm{M} ; 1$ cycle $7 \mu \mathrm{M},-$ (drug prepared in DMSO) invasion $38 \mu \mathrm{M}$ (DMSO); in cycle $12 \mu \mathrm{M} ; 1$ cycle assay $7 \mu \mathrm{M}$, Fig. 3a, Table 1). These results suggest that azithromycin can act independently against both merozoite invasion and intracellular parasite growth within one cycle of treatment in vitro.

To confirm that azithromycin was inhibiting merozoite invasion directly in our assays and was not acting downstream of invasion during intracellular parasite growth, the effect of exposure of merozoites or early ring-stage parasites to the drug was examined; azithromycin was washed out of the cultures within 1 hour and parasitaemia assessed by flow cytometry 40 hours postassay setup for all treatments (prior to the next round of parasite rupture and invasion of new host cells). 


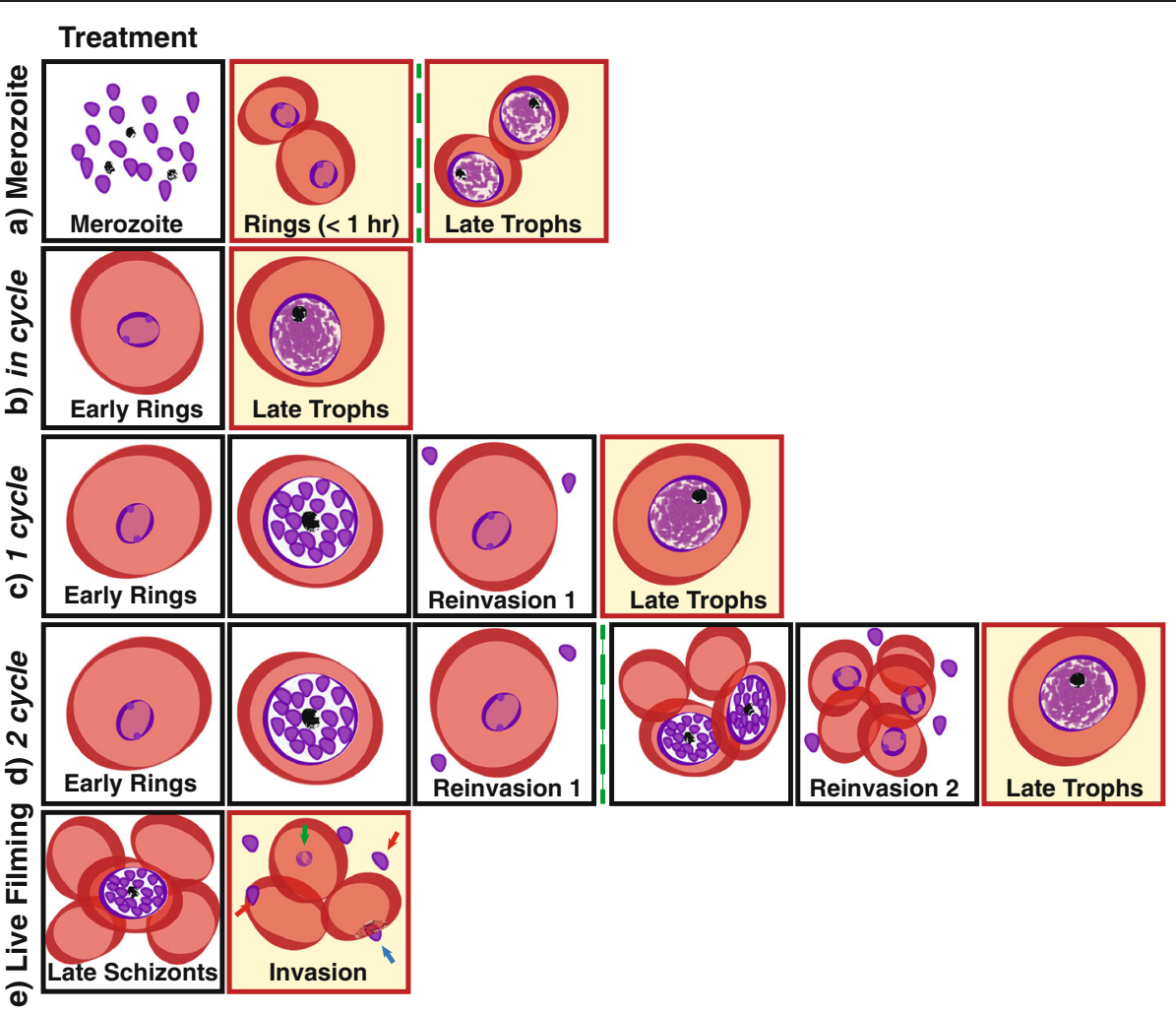

Fig. 1 Drug treatment strategies used in this study. The lifecycle stage of drug treatment is represented in the first box for each panel and the stage of parasitaemia measurements are highlighted by red boxes with yellow background. (a) Merozoite; successful invasion of drug-treated purified merozoites was measured at ring-stage immediately after addition of erythrocytes and merozoite invasion ( $<1$ hour rings) or after washing out the drug (denoted by green dashed line) and growing parasites through to late trophozoite stage (40 hours post-invasion). (b) In cycle; early ring-stage parasites ( $<4$ hours post-invasion) were drug-treated and the resulting growth inhibition was assessed at late trophozoite stage (40 hours post-invasion). (c) 1 cycle; early ring-stage parasites ( $<4$ hours post-invasion) were drug-treated and the resulting growth inhibition was assessed 90 hours later, after 1 cycle of reinvasion, at late trophozoite stage. These assays are a longer duration, but nonetheless equivalent in terms of including 1 cycle of reinvasion and development, to 1 cycle assays reported in other studies [41-44]. (d) 2 cycle (delayed death); early ring-stage parasites ( $<4$ hours post-invasion) were drug-treated and the drug-treated parasites were grown for 80 hours prior to washing out the drug in fresh media (denoted by green dashed line). Growth inhibition was assessed approximately 40 hours later after a second cycle of reinvasion (120 hours post-invasion). (e) Live filming; mature schizont-stage parasites were incubated with azithromycin and the success of merozoite invasion was recorded and quantified by live filming (green arrow, successful invasion; blue arrow, deformation of the erythrocyte but no invasion; red arrow, attachment and release or attachment with failure to deform erythrocyte and no release) (see Additional file 1: Video S1 and Additional file 2: Video S2). White box-drug treatment; yellow box-analysis of parasitaemia; green dashed line-drug washout

Treatment of merozoites (10 minute incubation) and early ring stages for less than 1 hour $(\mathrm{T}=0)$ with azithromycin $\left(1 \times \mathrm{IC}_{80}^{\text {mero }}\right.$ DMSO $\left.151 \mu \mathrm{M}\right)$ and the invasion inhibitory control compound, heparin, was significantly more inhibitory to parasite growth than the same treatment of early ring stages at the time-point of 20 minutes post-invasion ( $\mathrm{T}=20$ (exposed for 40 minutes); azithromycin $P<0.001$; heparin $P<0.01)$. Additionally, pretreatment of erythrocytes, followed by washout of drug, had little or no inhibitory effect on invasion (Fig. 3b; azithromycin $P<0.001$; heparin $P<0.001)$. This indicated that exposure of merozoites, but not very early ring stages or uninfected erythrocytes, was inhibitory to parasite growth.

In order to confirm that the inhibitory activity was against purified merozoites and not the uninfected erythrocyte prior to merozoite invasion, erythrocytes were pretreated with azithromycin at a $10 \times \mathrm{IC}_{80}^{\text {mero }}$ (380 $\mu \mathrm{M}$ (drug prepared in ethanol to limit nonspecific effects of vehicle)) prior to washing and addition of merozoites. There was minimal loss of invasion into erythrocytes pretreated with a $10 \times \mathrm{IC}_{80}^{\text {mero }}$ concentration of azithromycin for 1 hour relative to control (Fig. 3c). In contrast, merozoites treated for 10 minutes with the same concentration of azithromycin were unable to establish erythrocyte infections $(P<0.001)$, supporting the conclusion that it is the merozoite and its interaction with the erythrocyte, and not the erythrocyte itself, that is the target of inhibition.

Next we confirmed that azithromycin was inhibiting merozoite invasion directly, rather than acting on growth 
A

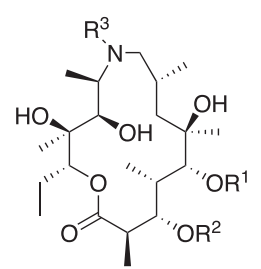

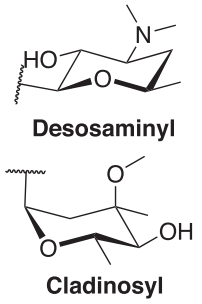

$1 \mathrm{R}^{1}=$ desosaminyl, $\mathrm{R}^{2}=$ cladinosyl, $\mathrm{R}^{3}=\mathrm{CH}_{3}$ (Azithromycin)

$2 \mathrm{R}^{1}=$ desosaminyl, $\mathrm{R}^{2}=\mathrm{H}, \mathrm{R}^{3}=\mathrm{CH}_{3}$ (Descladinosyl)

$3 R^{1}=H, R^{2}=H, R^{3}=\mathrm{CH}_{3}$ (Desglycan)

$4 \mathrm{R}^{1}=$ desosaminyl, $\mathrm{R}^{2}=$ cladinosyl, $\mathrm{R}^{3}=\left(\mathrm{CH}_{2}\right)_{2} \mathrm{NHCONH}\left(\mathrm{CH}_{2}\right)_{2} \mathrm{Ph}(\mathbf{1 2 e})$

$5 \mathrm{R}^{1}=$ desosaminyl, $\mathrm{R}^{2}=$ cladinosyl, $\mathrm{R}^{3}=\left(\mathrm{CH}_{2}\right)_{3} \mathrm{NHCONHCH}\left(\mathrm{CH}_{3}\right)$-1-naphthyl (1i)

$6 \mathrm{R}^{1}=$ desosaminyl, $\mathrm{R}^{2}=$ cladinosyl, $\mathrm{R}^{3}=\left(\mathrm{CH}_{2}\right)_{2} \mathrm{CONH}\left(\mathrm{CH}_{2}\right)_{2} \mathrm{Ph}$ (11c)

$7 \mathrm{R}^{1}=$ desosaminyl, $\mathrm{R}^{2}=$ cladinosyl, $\mathrm{R}^{3}=\left(\mathrm{CH}_{2}\right)_{3} \mathrm{NHCONH}\left(\mathrm{CH}_{2}\right)_{2}$-2-thiophenyl (7/47)

$8 \mathrm{R}^{1}=$ desosaminyl, $\mathrm{R}^{2}=$ cladinosyl, $\mathrm{R}^{3}=\left(\mathrm{CH}_{2}\right)_{3} \mathrm{NHCONH}-3,4$-dihydro-2H-benzo[b] $[1,4]$ dioxepine $(\underline{7 / 25})$

B

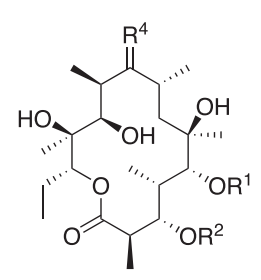

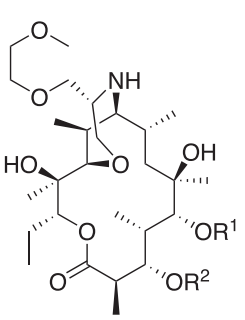

13 Dirithromycin

$\mathrm{R}^{1}=$ desosaminyl, $\mathrm{R}^{2}=$ cladinosy

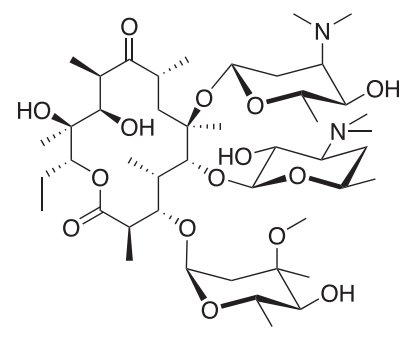

14 6-O-Megosaminyl erythromycin A

$9 \mathrm{R}^{1}=$ desosaminyl, $\mathrm{R}^{2}=$ cladinosyl, $\mathrm{R}^{4}=\mathrm{O}$ (Erythromycin)

$10 \mathrm{R}^{1}=$ desosaminyl, $\mathrm{R}^{2}=\mathrm{H}, \mathrm{R}^{4}=\mathrm{O}$ (Descladinosyl)

$11 \mathrm{R}^{1}=$ desosaminyl, $\mathrm{R}^{2}=$ cladinosyl, $\mathrm{R}^{4}=\mathrm{N}-\mathrm{OH}$ (Oxime)

$12 \mathrm{R}^{1}=$ desosaminyl, $\mathrm{R}^{2}=$ cladinosyl, $\mathrm{R}^{4}=\mathrm{N}-\mathrm{OCH}_{2} \mathrm{O}\left(\mathrm{CH}_{2}\right)_{2} \mathrm{OCH}_{3}$ (Roxithromycin)

C

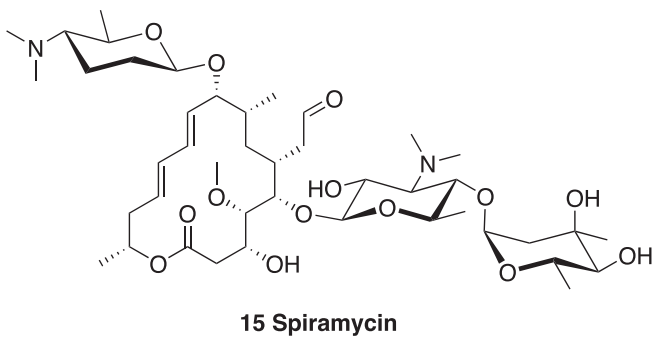

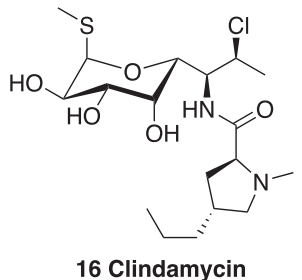

16 Clindamycin

Fig. 2 Structure of macrolide antibiotics. (a) Structure of the 15-membered macrolide, azithromycin, and its modified analogues (names used in the text underlined, in brackets). (b) Structure of the 14-membered macrolide, erythromycin A, and its modified analogues. (c) Structure of the 16-membered macrolide, spiramycin. (d) Structure of the non-macrolide antibiotic, clindamycin

downstream of invasion, through the complementary methods of microscopy and flow cytometric quantification of ring-stage parasites at the time-point of 1 hour postinvasion. There was an almost complete loss of ring-stage parasites seen by both microscopy and flow cytometry for azithromycin $\left(1 \times \mathrm{IC}_{80}^{\text {mero }}\right)$, confirming that azithromycin is indeed inhibitory to merozoite invasion (Fig. 3d,e,f). The merozoite invasion inhibitory activity of azithromycin was unaffected by limiting drug exposure to $<5$ seconds prior to erythrocyte addition, by the presence of serum in the culture medium or the presence of haemazoin crystals (that can remain after purification of merozoites) in the invasion assay. These results demonstrate that azithromycin is a rapid inhibitor of merozoite invasion in vitro.

\section{Azithromycin inhibits merozoite invasion prior to tight junction formation}

Live cell imaging of invading Plasmodium merozoites indicates that erythrocyte invasion is a multi-step process that typically takes less than 30 seconds to complete, after contact occurs between the merozoite and the erythrocyte, in primate species and slightly longer in 


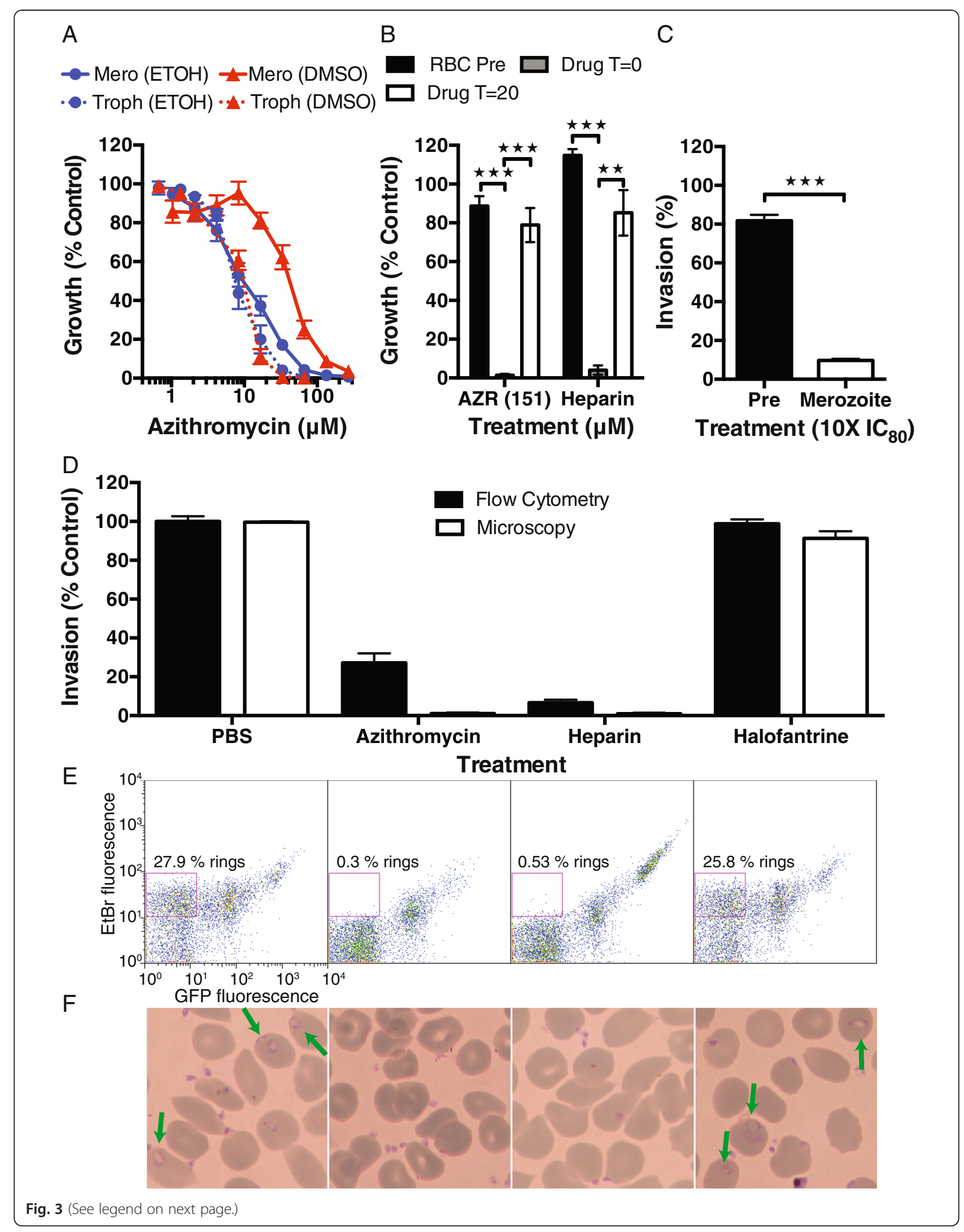


(See figure on previous page.)

Fig. 3 Azithromycin inhibits P. falciparum merozoite invasion. (a) The potency of azithromycin in ethanol or DMSO as vehicle was compared for invasion inhibition (unbroken line, 10 minute merozoite treatment, parasitaemia measured 40 hours later) and 1 cycle growth inhibition assays (broken line, treatment rings to trophozoites' next cycle). The invasion inhibitory $I C_{50}$ of azithromycin prepared in ethanol (blue, $\left.I C_{50} 10 \mu M\right)$ was similar to that for growth inhibition assays $\left(I_{50} 7 \mu \mathrm{M} ; P=0.0743\right.$, $\log I \mathrm{C}_{50}$ same between data sets, extra sum of squares $F$-test). The invasion inhibitory activity of azithromycin in DMSO (red, $\left.I C_{50} 38 \mu \mathrm{M}\right)$ was 5 -fold higher than 1 cycle growth assays $\left(I_{50} 7 \mu \mathrm{M} ; P<0.0001\right.$, Log $I C_{50}$ different between data sets). (b) Inhibition profiles for pretreated erythrocytes (RBC Pre), merozoite treatment ( $T=0$; drug added at time zero) and rings treated for $<1$ hour ( $T=20$; drug added 20 minutes post-invasion) were identical between azithromycin (in DMSO) and the invasion inhibitor heparin ( $\left(\mathrm{C}_{80}\right.$ concentration). (c) Increasing the concentration of azithromycin (in ethanol) to $10 \times I C_{80}(380 \mu M)$ did not result in substantial inhibition of invasion into pretreated cells compared to treatment of merozoites. (d) Flow cytometry and microscopy assessments confirmed that azithromycin $\left(I C_{80}\right.$ in ethanol) and heparin, but not the trophozoite-targeting antimalarial halofantrine $\left(2 \times I C_{80}\right.$ ring-stage treatment (46 nM) [13]), inhibit merozoite invasion and establishment of ring stages in erythrocytes. Representative (e) flow cytometry plots (GFP high and EtBr low ring-stage parasites represented by square gate) and (f) microscopy thin smears (rings highlighted by green arrows) show absence of ring-stage parasites for azithromycin and heparin compared to non-invasion inhibitory controls (labeling as per Fig. $3 \mathrm{~d}$ ). Experiments represent the mean and SEM of three or more experiments. Significance was tested using an unpaired t-test ${ }^{*} P=0.01-0.05,{ }^{* *} P \leq 0.01$, ***P $\leq 0.001)$

rodent species [35-37]. After contacting its target erythrocyte, the merozoite reorients its apical end onto the erythrocyte surface in a process that deforms the erythrocyte. This reorientation lasts about 10 seconds and is followed by the formation of a ring-like region of close and irreversible contact, called the tight (or moving) junction, between the merozoite apex and the erythrocyte. The merozoite enters the host cell through this junction using the power of its actin-myosin motor. After invasion, the erythrocyte surface reseals and the merozoite differentiates into a ring-stage parasite over several minutes [35]. We used live cell imaging to identify invasion step(s) that are inhibited by azithromycin (Fig. 1e). Late schizont-stage parasites were allowed to

Table 1 Growth/invasion inhibitory activity of compounds described in this study

\begin{tabular}{|c|c|c|c|c|c|c|c|}
\hline $\begin{array}{l}\text { Compound } \\
\text { number }\end{array}$ & $\begin{array}{l}\text { Compound } \\
\text { abbreviation }\end{array}$ & Compound name & $\begin{array}{l}\text { Lactone ring } \\
\text { size }\end{array}$ & $\| \mathrm{A} \mid \mathrm{C}_{50}(\mu \mathrm{M})$ & 40 hour $I_{50}(\mu \mathrm{M})$ & 90 hour $I C_{50}(\mu M)$ & 120 hour $I C_{50}(\mu M)$ \\
\hline 1 & $A Z R^{a}$ & Azithromycin & 15 & 10 & 6 & 7 & 0.04 \\
\hline 2 & AZR-desclad ${ }^{\mathrm{a}}$ & - & 15 & 50 & 39 & 31 & ND \\
\hline 3 & AZR-desglycan ${ }^{a}$ & - & 15 & $>1,600$ & $>500$ & 285 & ND \\
\hline 1 & $A Z R^{b}$ & Azithromycin & 15 & 38 & 12 & 7 & 0.1 \\
\hline 4 & $12 e^{b, c}$ & - & 15 & 15 & 1 & 0.6 & 0.16 \\
\hline 5 & $1 \mathrm{j}^{\mathrm{b}, \mathrm{c}}$ & - & 15 & 7 & 0.9 & 0.7 & 0.16 \\
\hline 6 & $11 c^{\mathrm{b}, \mathrm{d}}$ & - & 15 & 28 & ND & 0.9 & ND \\
\hline 7 & $7 / 47^{\mathrm{b}, \mathrm{e}}$ & - & 15 & 52 & ND & 5 & ND \\
\hline 8 & $7 / 25^{\mathrm{b}, \mathrm{e}}$ & - & 15 & 73 & ND & 10 & ND \\
\hline 9 & $\mathrm{ERY}^{\mathrm{a}}$ & Erythromycin & 14 & 420 & 230 & 52 & ND \\
\hline 10 & ERY-desclad ${ }^{a}$ & - & 14 & ND & 288 & 185 & ND \\
\hline 11 & ERY-oxime ${ }^{a}$ & - & 14 & 150 & 85 & 24 & ND \\
\hline 12 & $\mathrm{ROX}^{\mathrm{a}}$ & Roxithromycin & 14 & 83 & 70 & 27 & ND \\
\hline 13 & $D I R^{a}$ & Dirithromycin & 14 & 521 & 15 & 8 & ND \\
\hline 14 & Meg-ERYa,f & 6-0-megosaminyl-erythromycin & 14 & 13 & ND & ND & ND \\
\hline 15 & $\left.\mathrm{SP}\right|^{\mathrm{a}}$ & Spiramycin & 16 & 123 & 15 & 13 & ND \\
\hline 16 & $\left.C L\right|^{\mathrm{a}}$ & Clindamycin & NA & 743 & ND & ND & ND \\
\hline \multicolumn{8}{|c|}{ D10-AZR' (delayed death, resistant) comparison } \\
\hline 1 & $A Z R^{a}$ & Azithromycin & 15 & - & - & - & $0.16^{9, h}$ \\
\hline $1^{g}$ & $A Z R^{a}$ & Azithromycin & 15 & 25 & ND & ND & $9^{h}$ \\
\hline
\end{tabular}

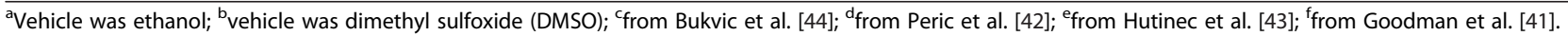
All IC $\mathrm{I}_{50}$ s are for the D10-PfPHG line used throughout this study, with the exception of ${ }^{9}$ which were undertaken with the D10 azithromycin-resistant line (D10-AZR ${ }^{r}$ ) made as per Goodman et al. [41]. All assays were measured by flow cytometry, with the exception of ${ }^{\mathrm{h}}$ which were measured by hypoxanthine uptake assays. Desclad, descladinosyl; desglycan, descladinosyl and desosaminyl; NA, not applicable; ND, not done 
rupture in the presence of 75 and $134 \mu \mathrm{M}$ azithromycin (in ethanol), which represent $2 \times \mathrm{IC}_{80}$ and $3.5 \times \mathrm{IC}_{80}$ of merozoite invasion inhibition concentrations, respectively. These slightly higher drug concentrations were used to clearly define the inhibitory phenotype. These concentrations were not found to inhibit schizont rupture. Five schizont ruptures were observed for each treatment. In the untreated control, 22 merozoites were observed to contact erythrocytes. After a contact period ranging from a few seconds to 2 minutes, $18 \%$ of the merozoites released or detached. A further $32 \%$ of the merozoites progressed to the erythrocyte deformation stage before releasing over a few minutes, and the remaining $50 \%$ advanced to complete invasion (Fig. 4). Azithromycin treatment at $75 \mu \mathrm{M}$ changed this profile with fewer merozoites invading (32\%) and a greater proportion failing to progress beyond the initial contact and deformation stages (68 \%, Fig. 4). At $134 \mu \mathrm{M}$ there was a significant and dramatic change in the invasion profile compared to the untreated control and azithromycin treatment at $75 \mu \mathrm{M}$ with no merozoites invading and most releasing after initial contact ( $81 \%$, Fig. 4). The observation that merozoites failed to make sustained contact with erythrocytes in the presence of azithromycin indicates that azithromycin acts early in invasion to prevent tight junction formation $[23,35,38]$. The periods of time that each of the invasion steps took to occur was also measured, but in nearly all cases showed no significant difference between the treatments. Representative video files showing the effects of azithromycin are shown in Additional file 1: Video S1 and Additional file 2: Video S2.

\section{Macrolides related to azithromycin also inhibit merozoite invasion}

After identifying that azithromycin was inhibitory to merozoite invasion, we examined whether related drugs with a history of clinical use as antibiotics also had this property. Macrolides with a 14-membered macrolactone ring (erythromycin $\mathrm{A}$, roxithromycin, dirithromycin) and 16-membered ring (spiramycin) were tested using the same methods as azithromycin (prepared in ethanol, Fig. 2b,c). Determination of the inhibitory concentration (Fig. 5a,b,c,d; Table 1) indicated that azithromycin (ethanol, $10 \mu \mathrm{M})$ had $>8$-fold lower $\mathrm{IC}_{50}$ for merozoite invasion when compared to roxithromycin $(83 \mu \mathrm{M})$ and spiramycin $(123 \mu \mathrm{M})$, while erythromycin $(420 \mu \mathrm{M})$ was 42 -fold less potent than azithromycin. Of interest was the very poor invasion inhibitory activity of dirithromycin $(521 \mu \mathrm{M})$, which was 52 -fold less potent than azithromycin, even though the $\mathrm{IC}_{50}$ values determined using 1 cycle growth assays were very similar (dirithromycin, $\mathrm{IC}_{50}^{90 h r} 8 \mu \mathrm{M}$; azithromycin, $\mathrm{IC}_{50}^{90 h r} 7 \mu \mathrm{M}$; Table 1). Invasion inhibition was confirmed by measurement of ring-stage parasites 1 hour after invasion at $1 \times \mathrm{IC}_{80}^{\text {mero }}$ for erythromycin $\mathrm{A}$, roxithromycin and spiramycin. The lack of inhibitory activity when erythrocytes were pretreated with drug (and then washed) further confirmed specific inhibition of merozoite invasion (Fig. 5e; $P<0.01)$.

Merozoite invasion inhibition is independent of macrolide activity against apicoplast ribosomes

Since macrolide antibiotics are known to target the 50S ribosomal subunit of the apicoplast to inhibit subsequent

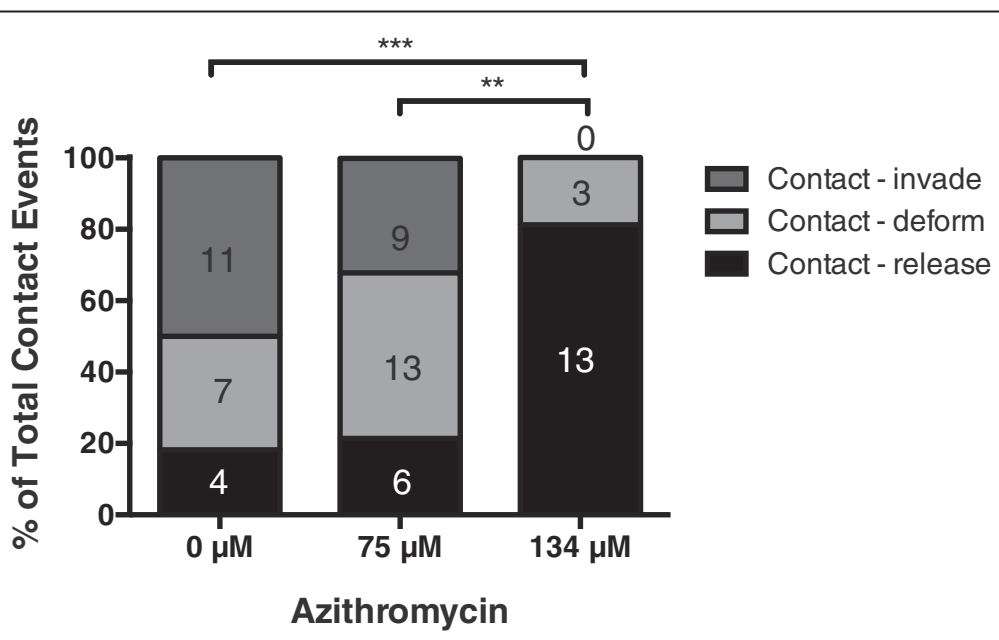

Fig. 4 Azithromycin inhibits the early steps of invasion. Video microscopy of merozoite invasion of erythrocytes was performed in the presence of 75 and $134 \mu \mathrm{M}$ azithromycin (in ethanol) compared to a no drug control $(0 \mu \mathrm{M})$. Five schizont ruptures were observed for each treatment. Of the merozoites that contacted erythrocytes, some were observed to deform erythrocytes and then successfully invade their host cells (contact-invade), while others did not progress beyond initial attachment (contact-detach) or progressed to deformation but did not invade (contact-deform). From several rupturing schizonts, the number of merozoites exhibiting each of these steps was counted for each drug treatment and the percentages are shown along with the number of events in the column boxes. A Chi-squared test was performed to indicate significant differences at the following levels (**P $\left.\leq 0.01,{ }^{* *} P \leq 0.001\right)$ 
intra-erythrocytic development, we tested the invasion inhibitory activity of clindamycin, which has a similar mechanism of action and exhibits overlapping binding of the $50 \mathrm{~S}$ ribosomal subunit of the apicoplast to azithromycin [39] (Table 1; Fig. 2c). Clindamycin had very weak invasion inhibitory activity; there was evidence for invasion inhibitory activity with a 74-fold higher $\mathrm{IC}_{50}$ observed for clindamycin (IC $\mathrm{I}_{50}^{\text {mero }} 743 \mu \mathrm{M}$ ) than seen for azithromycin $(10 \mu \mathrm{M})$. However, pre-treatment of erythrocytes with $2,972 \mu \mathrm{M}$ of clindamycin $\left(\mathrm{IC}_{80}^{\text {mero }}\right.$ ) resulted in a $65 \%$ reduction in merozoite invasion relative to untreated controls and there was evidence of erythrocyte lysis using these high levels of clindamycin (Fig. 6a). This suggests that at such high concentrations clindamycin is having a largely non-specific effect on merozoite invasion through damaging erythrocytes.

We tested further whether apicoplast ribosomal protein translation was the target of the inhibitory activity at the merozoite stage by comparison of the invasion inhibitory activity of azithromycin, erythromycin and clindamycin between the D10-PfPHG (azithromycinsensitive) parasite used throughout this study and an
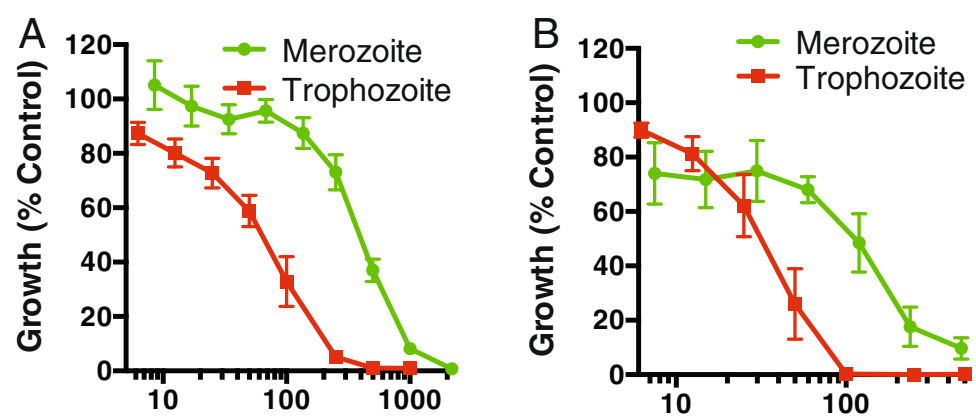

Erythromycin $(\mu \mathrm{M})$

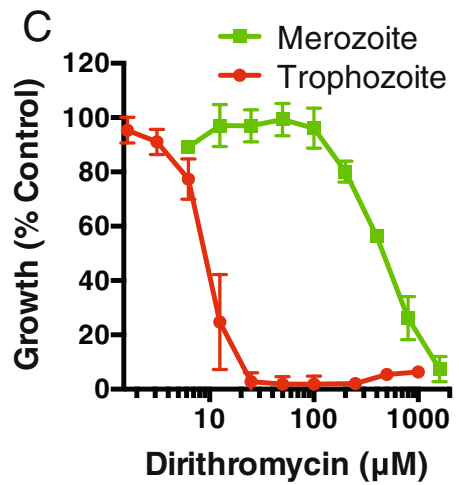

Roxithromycin $(\mu \mathrm{M})$
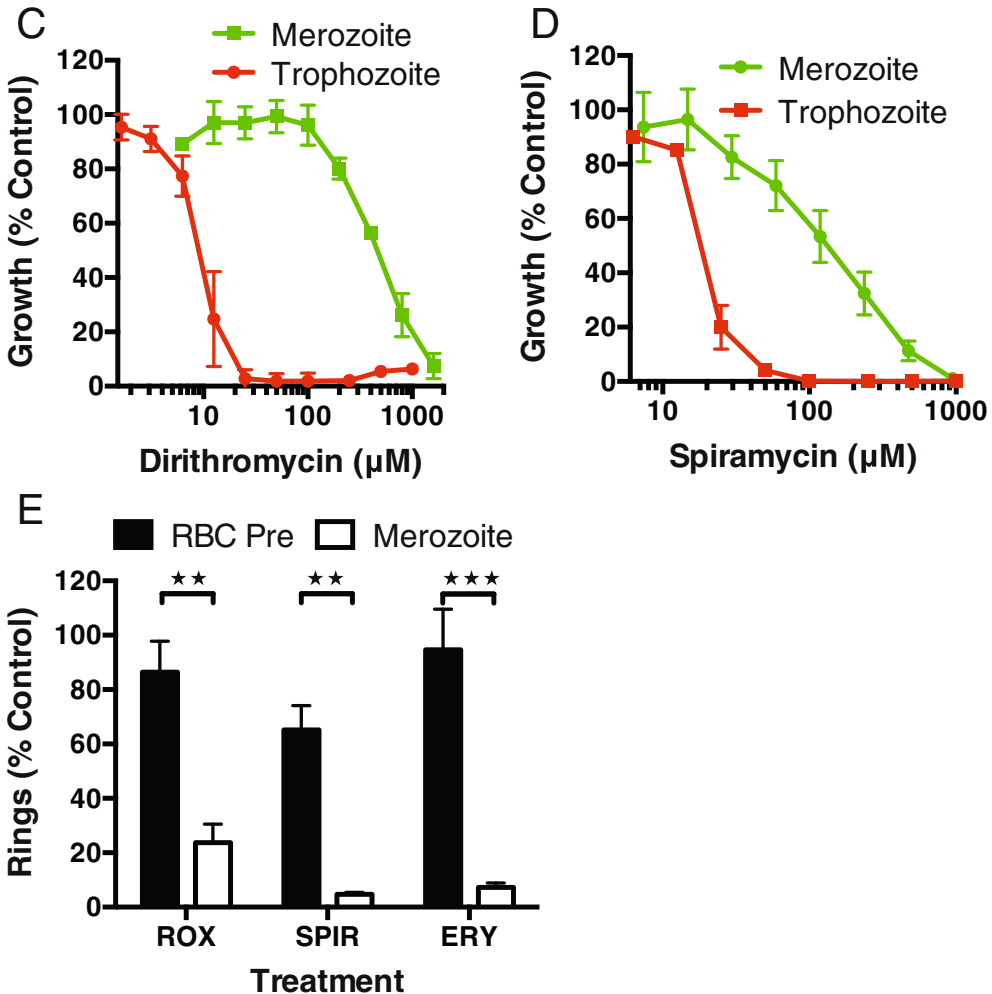

Fig. 5 Related macrolides inhibit merozoite invasion. The 14-membered macrolides (a) erythromycin $A\left(I C_{50}^{\text {mero }} 420 \mu M\right)$, (b) roxithromycin ( $\left.83 \mu M\right)$, (c) dirithromycin $(521 \mu \mathrm{M})$ and $(\mathbf{d})$ the 16-membered macrolide spiramycin $(123 \mu \mathrm{M})$ had variable levels of invasion inhibitory activity (green) and a higher $\mathrm{IC}_{50}$ than that achieved for 1 cycle assays (red). (e) The invasion inhibitory activity of erythromycin A, roxithromycin and spiramycin at an $I_{80}$ concentration was confirmed by flow cytometry assessment of ring stages with minimal inhibition evident for pretreated erythrocytes. All experiments represent the mean and SEM of three or more experiments. Significance of differences was compared using an unpaired t-test ( ${ }^{* *} P \leq 0.01$, *** $P \leq 0.001$ ) 
azithromycin-resistant D10 derivative (D10-AZR ${ }^{\mathrm{r}}$ ) selected for reduced sensitivity to both azithromycin and erythromycin in 2 cycle (delayed death) assays. Sequencing of apicoplast ribosome genes revealed a G91D mutation in the rpl4 gene product, corresponding to the G112D mutation in Chlamydomonas reinhardtii, known to confer resistance to erythromycin [40]. This mutation is associated with a 57 -fold loss of sensitivity to azithromycin over 2 cycles of parasite growth ( $\mathrm{IC}_{50}^{120 h r} \mathrm{D} 10$ $\mathrm{AZR}^{\mathrm{r}}, 9 \mu \mathrm{M}$; D10 parental, $0.16 \mu \mathrm{M} ; P<0.0001$; Fig. 6b; Table 1). Comparison of the $\mathrm{IC}_{50}^{\text {mero }}$ (Fig. 6c,d,e) suggested that there was very little difference in merozoite invasion inhibitory activity between the resistant versus sensitive lines for azithromycin (in ethanol, 2.5-fold difference; $\mathrm{IC}_{50}$ D10-AZR ${ }^{\mathrm{r}}, 25 \mu \mathrm{M}$; D10-PfPHG, $10 \mu \mathrm{M}$; $P<0.0001$ ), erythromycin (1.6-fold difference; $\mathrm{IC}_{50} \mathrm{D} 10$ $\mathrm{AZR}^{\mathrm{r}}, 732 \mu \mathrm{M}$; D10-PfPHG, $\left.442 \mu \mathrm{M} ; P=0.0247\right)$ or clindamycin (1.3-fold difference; $\mathrm{IC}_{50}$ D10-AZR ${ }^{\mathrm{r}}, 556$ $\mu \mathrm{M}$; D10-PfPHG, $736 \mu \mathrm{M} ; P=0.26$ ). Together, these data suggest that the invasion inhibitory activity of azithromycin is largely independent of apicoplast ribosomal protein synthesis, consistent with the current view that the apicoplast does not play a role in invasion.

\section{Modification of macrolides enhances invasion inhibitory activity}

We tested a panel of macrolide analogues to determine whether the $\mathrm{IC}_{50}$ of merozoite invasion inhibition could be reduced. An erythromycin A L-megosamine sugar derivative (Meg-erythromycin, 6-O-megosaminyl erythromycin A) that has a lower $\mathrm{IC}_{50}$ in 1 cycle growth inhibition assays [41] was tested and found to reduce the $\mathrm{IC}_{50}$ of merozoite invasion inhibition $(13 \mu \mathrm{M}) 32$-fold compared to the parent compound $(420 \mu \mathrm{M}$; Fig. 7a; Table 1; Fig. 2b). Furthermore, addition of an oxime group $(\mathrm{N}-\mathrm{OH})$ to erythromycin A (erythromycin oxime) lowered the invasion inhibitory $\mathrm{IC}_{50}(150 \mu \mathrm{M})$ almost 3 -fold compared to erythromycin A. The increased potency of Meg-erythromycin and erythromycin oxime compared to the parent drug indicates that various modifications to macrolides can lead to more potent invasion inhibitory activity.

Next we obtained a small panel of azithromycin analogues (in DMSO) from GlaxoSmithKline (Tres Cantos, Spain) [42-44] with reported $\mathrm{IC}_{50}$ values lower than azithromycin in a 72 hour growth inhibition assay (roughly equivalent to 1 cycle assays in this study; Fig. 1c). In our studies, we found that azithromycin dissolved in DMSO was less effective so the following comparisons use the $\mathrm{IC}_{50}$ values for both azithromycin and the azithromycin analogues with DMSO as the solubilization vehicle. Two compounds (12e, $15 \mu \mathrm{M} ; 1 \mathrm{j}, 7 \mu \mathrm{M})$ had a substantially lower $\mathrm{IC}_{50}$ for merozoite invasion inhibition than azithromycin (38 $\mu \mathrm{M}$ (when prepared in DMSO); Fig. 7b;
Table 1; Fig. 2a), indicating that azithromycin can also be modified to lower its invasion inhibitory activity in vitro.

When early ring-stage parasites were treated with the compounds $1 \mathrm{j}$ and $12 \mathrm{e}$ for 40 hours (in cycle; $1 \mathrm{j}, 0.9 \mu \mathrm{M}$; $12 \mathrm{e}, 1 \mu \mathrm{M})$ and 90 hours (1 cycle; $1 \mathrm{j}, 0.7 \mu \mathrm{M} ; 12 \mathrm{e}$, $0.6 \mu \mathrm{M})$ the $\mathrm{IC}_{50}$ of the modified analogues was between 10- and 14-fold lower than that of azithromycin (DMSO; in cycle, $12 \mu \mathrm{M}$; 1 cycle, $7 \mu \mathrm{M}$; Fig. 7c; Table 1). In contrast, parasites treated for 120 hours (2 cycle; delayed death, Fig. 1d) showed a small increase in the potency of azithromycin (DMSO, $0.1 \mu \mathrm{M}$ ) over $1 \mathrm{j}$ $(0.16 \mu \mathrm{M})$ and $12 \mathrm{e}(0.16 \mu \mathrm{M})$ for the 'delayed death' inhibition typical of apicoplast ribosome targeting (Table 1). This would suggest that the apicoplast ribosome targeting is not affected by the modifications of $1 \mathrm{j}$ and $12 \mathrm{e}$, and is a further indication that invasion inhibition or parasite growth inhibition over 1 cycle of parasite growth or less is not a result of inhibition of apicoplast ribosome activity. Further, the ability of azithromycin and its analogues to inhibit intracellular parasite growth during in cycle assays (from early rings to late trophozoites) at $\mathrm{IC}_{50}$ values lower than the invasion inhibition assays suggests that the compounds can inhibit parasite growth independent of merozoite invasion.

In order to assess whether the invasion inhibitory phenotype of a more potent analogue was similar to our observations for azithromycin in ethanol (see Fig. 4b), live filming of merozoite invasion in the presence of analogue $12 \mathrm{e}$ was examined using live video microscopy. As was observed in Fig. 4, for the no drug treatment control $53 \%$ of merozoites that contacted an erythrocyte invaded, 29 \% deformed but failed to invade the erythrocyte after contacting and $18 \%$ released the erythrocyte membrane without invading or deforming the erythrocyte (Fig. $7 \mathrm{~d})$. In contrast, addition of $12 \mathrm{e}$ at $122 \mu \mathrm{M}\left(2 \times \mathrm{IC}_{80}\right)$ reduced invasion to only $8 \%$ of total contact events, reduced contacts leading to erythrocyte deformation to 27 $\%$ of contact events and increased the number of merozoites releasing without invading or deforming the erythrocyte to $65 \%$ of all contact events, resulting in failure to invade for $92 \%$ of all contact events. These results support the live filming observations for azithromycin in ethanol (Fig. 4b) and again indicate that azithromycin acts early in the invasion process and inhibits tight junction formation. We compared the invasion phenotype of $12 \mathrm{e}$ to that of azithromycin in DMSO at an equal concentration (approximately $1 \times \mathrm{IC}_{80}$ of azithromycin in DMSO). As expected, at the same concentration the less potent parent azithromycin was less inhibitory to invasion ( $25 \%$ contact events invaded) and as a result had fewer failed invasion events (75\%). Together, these findings establish a proof-of-concept that macrolides with more potent invasion inhibitory activity can be developed through 

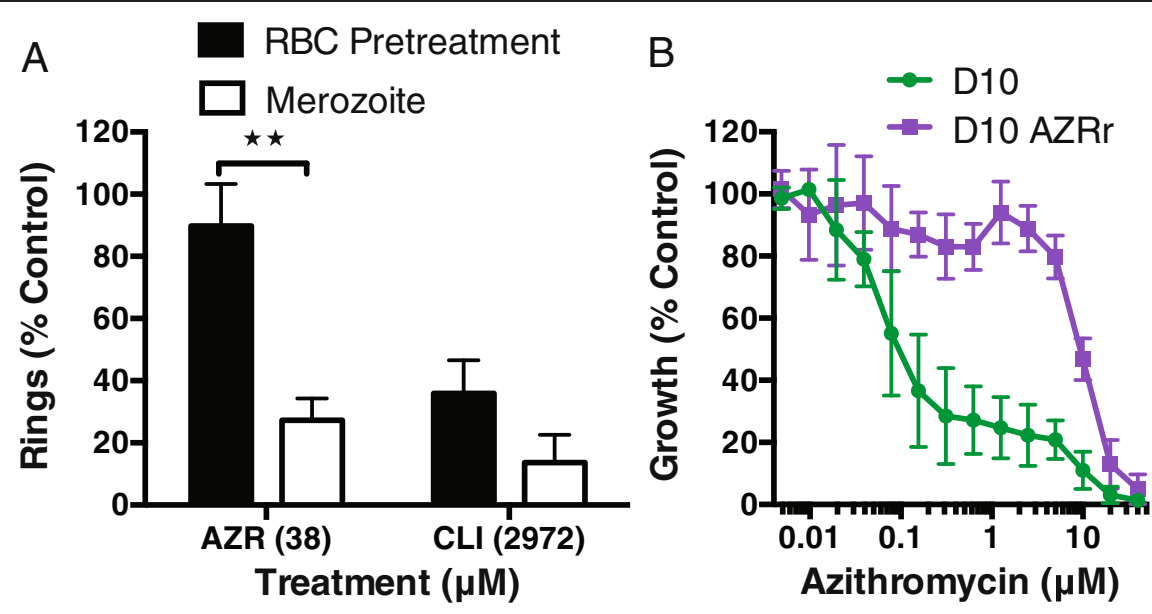

C

D
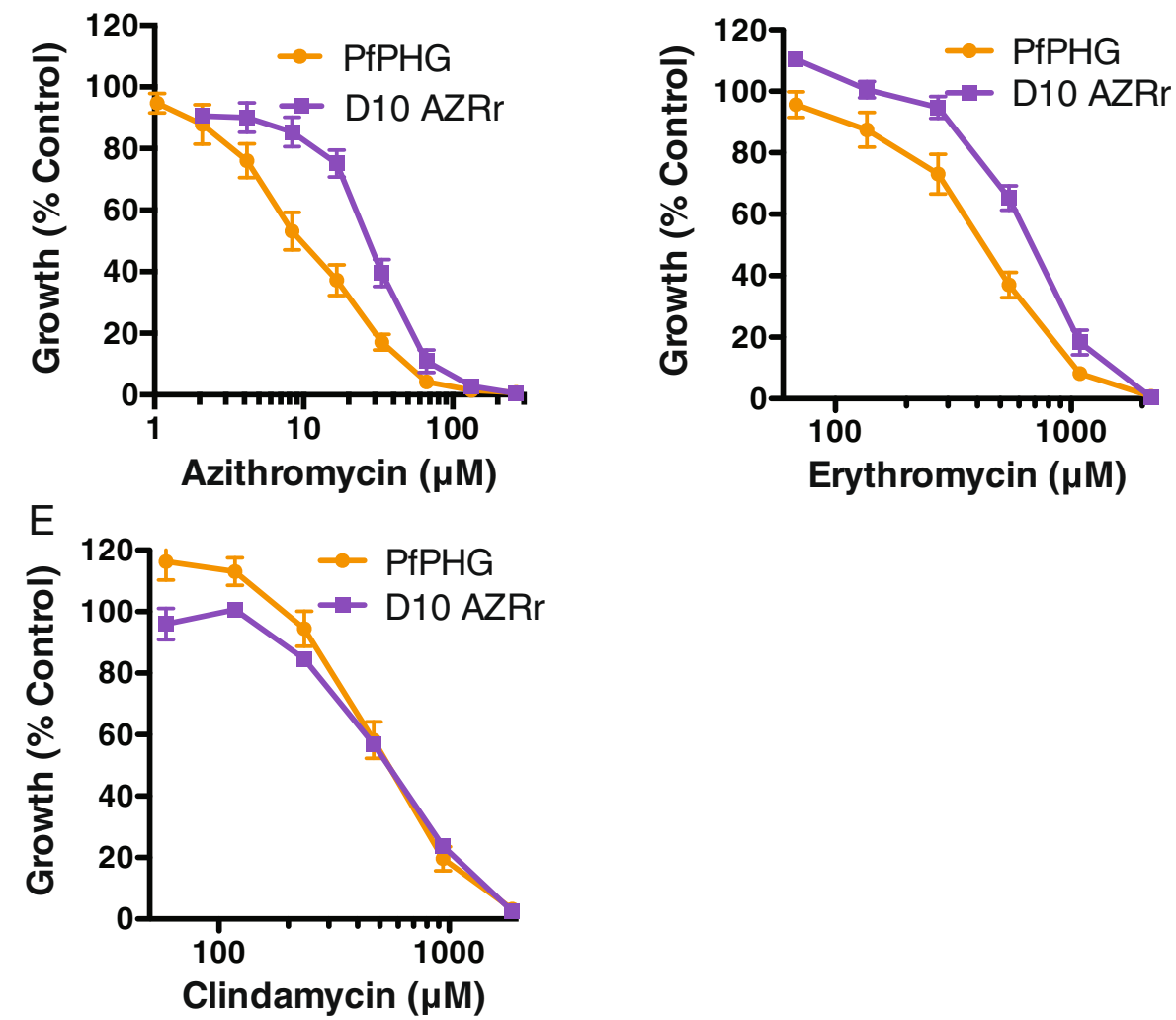

Fig. 6 The mechanism of invasion inhibition is unlikely to target the apicoplast ribosome. (a) Clindamycin targets the same subunit of the apicoplast ribosome but was found to have a much higher $\mathrm{IC}_{80}$ for apparent merozoite invasion inhibition $(2,972 \mu \mathrm{M})$. There was evidence of non-specific inhibition of invasion as pretreatment of erythrocytes with clindamycin gave significant inhibition, which was not seen for azithromycin (prepared in ethanol; mean and SEM of four or more experiments; significance of differences tested with an unpaired t-test; $\left.{ }^{* *} P \leq 0.01\right)(38 \mu M$ ). (b) The D10-AZR line showed up to a 57-fold higher tolerance of azithromycin in 2 cycle (delayed death) apicoplast-targeting drug inhibition assays compared to D10 parental line. In contrast, the $I C_{50}$ for purified merozoite invasion inhibitory activity differed by less than 2.5-fold between the D10-AZR line and the D10-PfPHG line for (c) azithromycin (IC 50 : PfPHG $10 \mu \mathrm{M}$; D10-AZR $25 \mu \mathrm{M}$ ), (d) erythromycin A (IC 50 : PfPHG 420 $\mu$ M; D10-AZR 732 $\mu$ M) and (e) clindamycin $\left(\mathrm{IC}_{50}\right.$ : PfPHG $743 \mu \mathrm{M}$; D10-AZR $\left.557 \mu \mathrm{M}\right)$. Data represent the mean of two or more experiments in at least duplicate. D10-AZR', D10 azithromycin-resistant

chemical modification and that the dual activities of invasion inhibition and other inhibitory activities can be developed in single compounds and are not mutually exclusive.
Identification of structural groups on azithromycin important for invasion inhibitory activity

We sought to identify structural groups of azithromycin that were most important for invasion inhibitory activity 

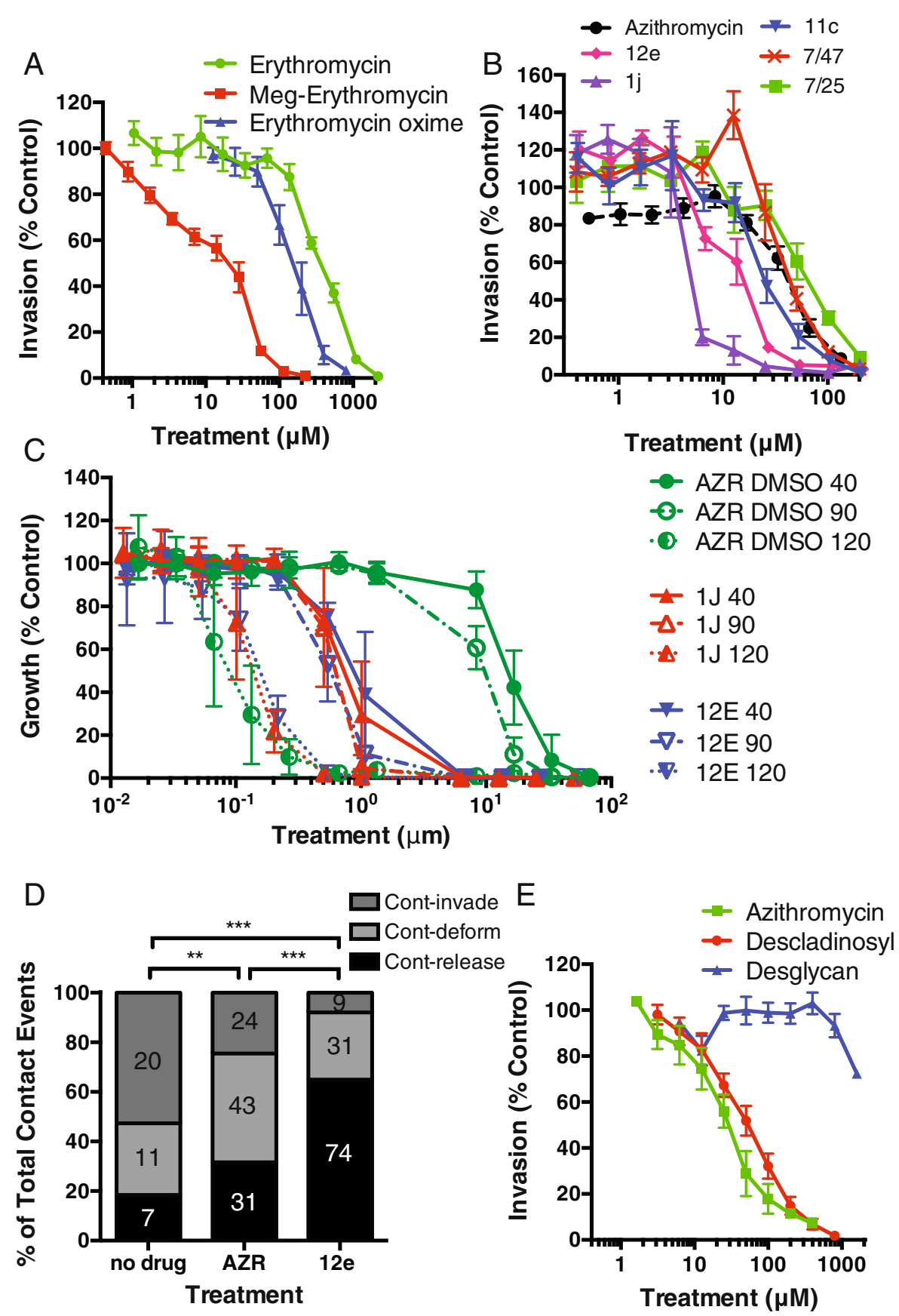

Fig. 7 Macrolide modification lowers invasion inhibitory $I_{50}$, but not apicoplast-targeting 'delayed death' inhibition. (a) Addition of an L-megosamine sugar [41] to form Meg-erythromycin (6-O-megosaminyl erythromycin $A$, IC $C_{50} 13 \mu \mathrm{M}$ ) or an oxime group (150 $\left.\mu \mathrm{M}\right)$ lowered the invasion inhibitory $I C_{50}$ activity compared to the parent drug erythromycin A ( $\left.\mathrm{I}_{50} 420 \mu \mathrm{M}\right)$. (b) Screening of an azithromycin analogue panel identified three compounds (12e, $15 \mu \mathrm{M} ; 1 \mathrm{j}, 7 \mu \mathrm{M} ; 11 \mathrm{c}, 28 \mu \mathrm{M}$ ) with up to 5-fold lower invasion inhibitory $\mathrm{IC}_{50}$ compared to the parent azithromycin (in DMSO, $38 \mu \mathrm{M}$ ). (c) Treatment of parasites during in cycle (40 hours, rings to schizonts), 1 cycle (90 hours, 1 cycle of replication) and 2 cycle (120 hours, 2 cycles of replication) assays with azithromycin (in DMSO) and analogues (12e, 1j), indicated that the $I_{50}$ of 1 cycle (40 hour and 90 hour, high drug concentration) inhibition was greatly reduced for the analogues compared to azithromycin. In contrast, the $I_{50}$ of the delayed death phenotype was almost identical for azithromycin and its analogues. d) Video microscopy of merozoite invasion was performed in the presence of a no drug control $(0 \mu \mathrm{M}), 122 \mu \mathrm{M}$ of analogue 12e $\left(2 \times I_{80}\right)$ and azithromycin (AZR; both in DMSO). Merozoites that contacted the erythrocyte and i) invaded (cont-invade), ii) deformed but did not invade (cont-deform), or iii) released without deforming or invading (cont-detach) were tallied and analyzed as per Fig. 4. (e) Removal of the cladinosyl sugar (azithromycin descladinosyl, $50 \mu \mathrm{M}$ ) from azithromycin increased the invasion inhibitory $I C_{50}$ compared to azithromycin $(10 \mu \mathrm{M})$. The additional removal of the desosaminyl sugar (azithromycin desglycan, $I C_{50}>1,600 \mu M$ ) resulted in loss of invasion inhibitory activity compared to azithromycin. Data represent the mean and SEM of three or more experiments $\left({ }^{* *} P \leq 0.01,{ }^{* * *} P \leq 0.001\right)$ 
by determining the effect of removing one or both of the glycosylated groups. The two glycan groups of azithromycin, in particular the desosamine group, have been identified as critical to proper drug binding and inhibition of bacterial ribosome translation [39]. Removal of the cladinosyl group (descladinosyl) for both azithromycin and erythromycin A led to an increase of the drugs $\mathrm{IC}_{50}$ for both in cycle (azithromycin, $6 \mu \mathrm{M}$, AZRdescladinosyl, $39 \mu \mathrm{M}$; erythromycin A, $230 \mu \mathrm{M}$, ERYdescladinosyl, $288 \mu \mathrm{M}$ ) and 1 cycle (azithromycin, $7 \mu \mathrm{M}$; AZR-descladinosyl, $31 \mu \mathrm{M}$; erythromycin A, $52 \mu \mathrm{M}$; ERY-descladinosyl, $185 \mu \mathrm{M}$ ) drug assays (Table 1; Fig. 2a,b). Similarly, removal of the cladinosyl group from azithromycin (AZR-descladinosyl, $50 \mu \mathrm{M}$ ) led to 5 -fold reduction in the invasion inhibitory activity of the compound compared to azithromycin (azithromycin, $10 \mu \mathrm{M}$, Fig. 7e).

We were further able to remove both the cladinosyl and desosamine groups from azithromycin (AZR-desglycan; Fig. 7e; Table 1; Fig. 2a), which led to a dramatic loss of invasion inhibitory activity and an increase in the $\mathrm{IC}_{50}$ beyond the limits of the assay $(>1,600 \mu \mathrm{M})$. Comparison of these compounds indicates that the cladinosyl group has a role in lowering the $\mathrm{IC}_{50}$ of invasion inhibition for azithromycin, but the presence of both the cladinosyl and desosamine groups is critical for azithromycin's invasion inhibitory activity. While these data suggest that the desosamine group plays the critical role during invasion inhibition, technical limitations prevented the selective removal of the desosamine group.

\section{Azithromycin inhibits $P$. berghei merozoite and Toxoplasma gondii tachyzoite invasion, but not $P$. berghei sporozoite invasion}

To test whether azithromycin could inhibit invasion of $P$. berghei, widely used as a murine model of malaria, we purified merozoites [45] and allowed invasion to proceed in the presence of azithromycin. Azithromycin inhibited merozoite invasion of $P$. berghei at very similar concentrations to that seen for $P$. falciparum, with no evidence of inhibition for parallel treatments of newly invaded rings (Fig. 8a,b).

Since azithromycin was found to be an effective inhibitor of merozoite invasion, we tested whether this drug could also inhibit invasion of $P$. berghei sporozoites into liver cells in vitro. The effects of azithromycin on $P$. berghei in vitro liver-stage infections following invasion have been previously reported to be limited to retardation of apicoplast development with no effect on overall parasite growth (up to 65 hours) [46]; the effect of azithromycin on sporozoite invasion has not directly been examined. Initially, we assayed the ability of $P$. berghe $i$ sporozoites to enter liver cells in vitro using an established assay. GFP-expressing sporozoites were allowed to settle on and invade HepG2 cells. After free sporozoites were removed by washing, cells were fixed and then antibody-labeled without cell permeabilization, thereby preventing labeling of sporozoites that are inside host cells. We then compared the number of labeled cells (external) to the total number of cells (internal and external, as measured by GFP expression) to determine invasion efficiency. There was a statistically significant, but modest, effect indicating that azithromycin may inhibit sporozoite invasion of hepatocytes at concentrations up to $100 \mu \mathrm{M}$ azithromycin $(100 \mu \mathrm{M}, 42 \%$ reduction in cell entry; (azithromycin prepared in ethanol); Fig. 8c). However, this assay is unable to robustly distinguish between sporozoites traversing liver cells from those establishing an infection. Therefore, to assess the impact of azithromycin treatment on the establishment of successful infection of liver cells, we repeated the treatment and then allowed any successfully invaded sporozoites to develop for 24 hours; this is a point at which parasites developing within the liver cell can be distinguished from any remaining sporozoites that have not established infection. The number of parasites successfully establishing an infection and forming a vacuole at 24 hours did not differ between azithromycin-treated and control (ethanoltreated) cultures. This suggests that while there was some evidence of reduced sporozoite invasion of liver cells, this did not result in measurably reduced hepatocyte infections by $P$. berghei sporozoites (Fig. $8 \mathrm{~d}$ ).

We next explored whether azithromycin could inhibit invasion of the related apicomplexan parasite, Toxoplasma gondii, which has orthologues of many Plasmodium invasion proteins and a similar invasion process [47]. There was a significant dose-dependent inhibition of tachyzoite invasion at azithromycin concentrations (in ethanol) between $250 \mu \mathrm{M}$ and $50 \mu \mathrm{M}$ (Fig. 8e). Azithromycin inhibited $47 \%$ of tachyzoite invasions at the highest concentration tested $(250 \mu \mathrm{M})$ compared to $90 \%$ inhibition for the invasion inhibitory control cytochalasin D $(1 \mu \mathrm{M})$ [48] and the invasion inhibitory $\mathrm{IC}_{80}$ of azithromycin against $P$. falciparum merozoite ( $38 \mu \mathrm{M}$; in ethanol), clearly indicating that azithromycin is a less potent inhibitor of $T$. gondii invasion in vitro. Importantly, there was no evidence of tachyzoite invasion inhibition in the presence of 500 $\mu \mathrm{M}$ erythromycin A, reflecting the results of the $P$. falciparum studies which showed that erythromycin A is a poor inhibitor of merozoite invasion compared to azithromycin. We next explored whether the azithromycin analogue $12 \mathrm{e}$, which was found to be a more potent inhibitor of $P$. falciparum merozoite invasion than azithromycin (DMSO as vehicle), had improved tachyzoite invasion inhibitory activity compared to azithromycin. Analogue 12e (tested at $42 \mu \mathrm{M}$ due to limited compound availability) was found to be a consistently 

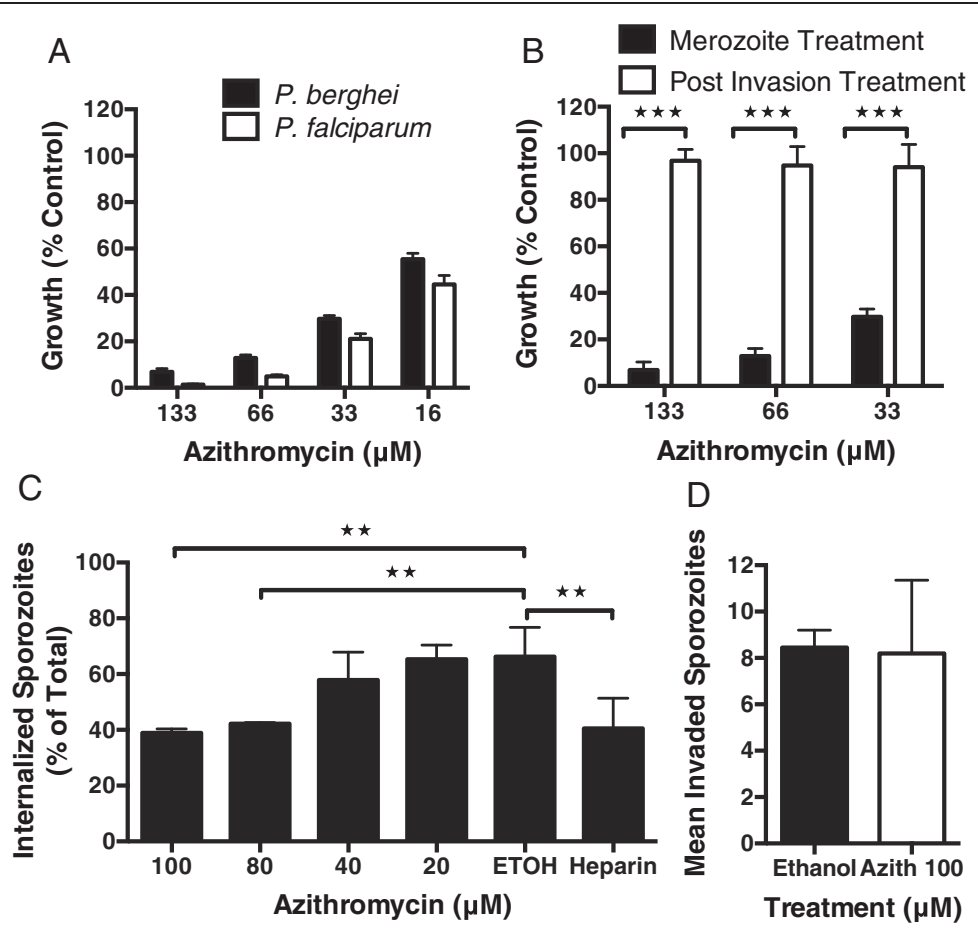

D
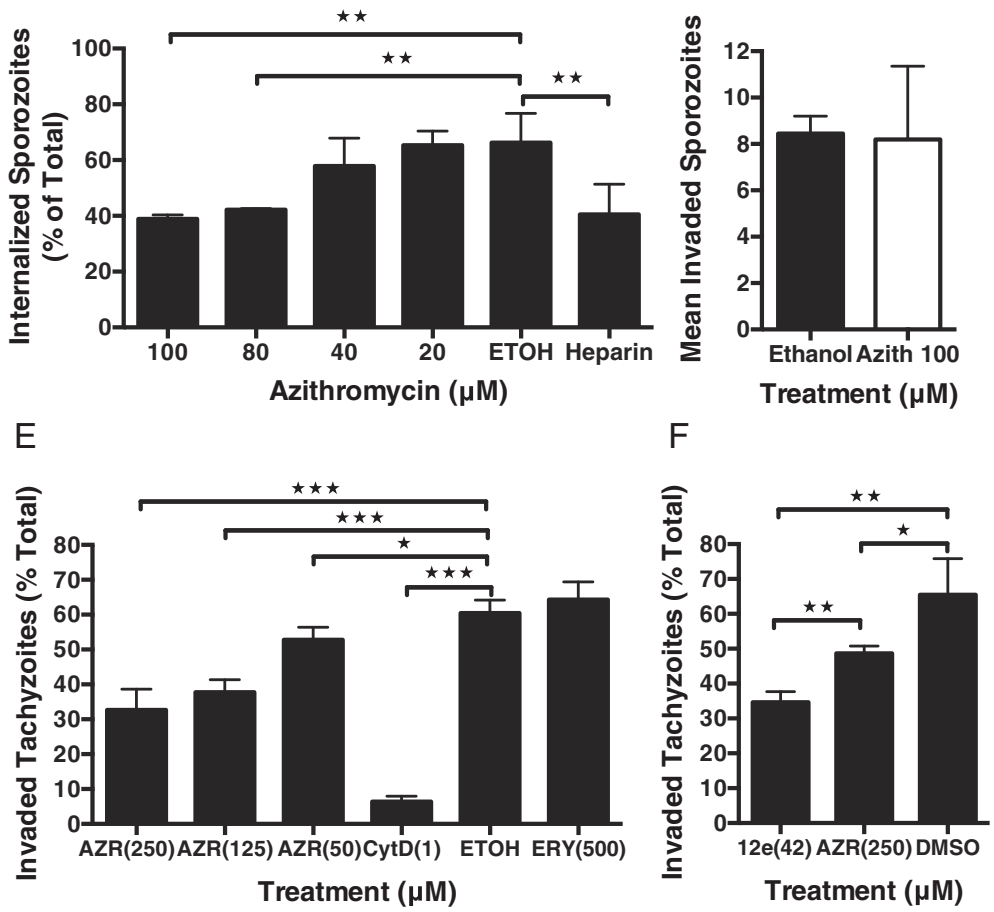

Fig. 8 Azithromycin inhibits P. berghei merozoite and Toxoplasma gondii tachyzoite, but not sporozoite, invasion of host cells. (a) Azithromycin inhibited purified $P$. berghei merozoite invasion at similar concentrations compared to $P$. falciparum. (b) Treatment of $P$. berghei-infected erythrocytes immediately after invasion with azithromycin for 30 minutes did not result in a loss of late-stage parasites detected by flow cytometry, confirming that azithromycin inhibited invasion and not parasite growth. (c) Azithromycin was found to have a small but significant effect on the number of $P$. berghei sporozoites that had entered or traversed host cells. However, using a more specific assay that measures successfully invaded hepatocytes containing a developing parasite (d), there was no significant inhibition in sporozoites that had invaded and formed a parasitophorous vacuole. (e) Azithromycin treatment of $T$. gondii tachyzoites at concentrations of 250, 125 and $50 \mu \mathrm{M}$ resulted in a dose-dependent inhibition of host cell invasion. The invasion inhibitory control cytochalasin D $(1 \mu \mathrm{M})$ was a considerably more potent inhibitor of tachyzoite invasion, while erythromycin $\mathrm{A}$, as was found for $P$. falciparum, showed no evidence of invasion inhibitory activity at concentrations up to $500 \mu \mathrm{M}$. (f) Azithromycin analogue 12e (42 $\mu \mathrm{M}$ ) was significantly more inhibitory to Toxoplasma tachyzoite invasion than azithromycin ( $250 \mu \mathrm{M}$, both solubilized in DMSO) even when tested at a 6 -fold lower concentration. Data represent the mean and SEM of three or more experiments, significance of differences was tested using an unpaired t-test $\left({ }^{*} P=0.01\right.$ to $0.05,{ }^{* *} P \leq 0.01,{ }^{*}{ }^{*} P \leq 0.001$ )

more potent inhibitor of $T$. gondii tachyzoite invasion (48\% inhibition compared to non-inhibitory control) than much higher concentrations of azithromycin (250 $\mu \mathrm{M}, 24 \%$ inhibition, Fig. 8e), suggesting that analogues of azithromycin could be developed to have much greater potency against $T$. gondii host cell invasion than the parent compound. These data suggest that azithromycin and analogues are effective inhibitors of Plasmodium spp. merozoite and T. gondii tachyzoite invasion, raising the possibility that azithromycin may target proteins or events conserved between organisms. However, there are clearly differences in drug potency between parasites hence drugs will have to be optimized to target invasion for each organism. 


\section{Discussion}

Successful control and treatment strategies for malaria currently rely on the availability of effective antimalarial drugs. The emergence of resistance to the critically important artemisinin family of drugs [7-9] highlights the need for new drugs to partner and replace current therapies, and new drug development strategies. Drugs that target merozoite invasion have been proposed as a novel strategy for antimalarial development [11-14]. We have applied robust methods for the purification of viable P. falciparum merozoites and invasion assays $[13,18]$ to screen for inhibitors of merozoite invasion. The antibiotic azithromycin and related compounds were identified as rapid inhibitors of $P$. falciparum merozoite invasion into erythrocytes. This is the first time that an antimalarial in clinical use has been linked to inhibition of merozoite invasion in vitro, and the first clear identification of antimalarial compounds with dual mechanisms of action against merozoite invasion and intraerythrocytic parasite development. Live video microscopy revealed that azithromycin acts to prevent the essential step of tight junction formation during invasion. Furthermore, the inhibitory activity of azithromycin was largely ablated by selective removal of glycan groups, and we identified modified macrolides with increased potency against merozoite invasion.

Azithromycin is a macrolide antibiotic with a 15membered macrolactone ring that is well tolerated and safe for clinical use by children and pregnant women [28]. Azithromycin and related macrolides are known to inhibit ribosomal protein synthesis in the asexual-stage parasite apicoplast by binding to the $50 \mathrm{~S}$ subunit of the apicoplast (70S) ribosomal complex [49, 50]. Typically, clinical concentrations of azithromycin based on current dosing regimens result in 'delayed death' of the parasite in in vitro assays. The progeny of azithromycin-treated parasites fail to form functional apicoplasts in the daughter merozoites, thereby causing the death of the second generation of parasites after treatment [31, 32]. Azithromycin has been considered a candidate for inclusion in artemisinin combination therapies (ACT) and for the prevention of malaria in pregnancy [51] due to its safety and its long half-life (over 50 hours) [25, 27, 29].

In addition to its 'delayed death' effect on parasite growth, azithromycin has been reported to kill parasites within the first cycle of in vitro parasite growth at a drug concentration just above reported peak plasma concentrations $[29,32]$. It has been speculated that inhibition of parasite growth during the first cycle of treatment is a result of azithromycin having a secondary target in addition to the apicoplast ribosome [32, 41]. Given growth inhibition via apicoplast-targeting 'delayed death' is approximately 150 -fold more potent than that achieved for 1 cycle growth inhibition in vitro, azithromycin is thought to act predominantly through targeting the apicoplast when used clinically. Because of azithromycin's safety profile, several studies have investigated modifications to the drug with a view to lowering the $\mathrm{IC}_{50}$ of short-term treatment (1 cycle of growth) to the low nanomolar range [4244]. Yet despite this interest in azithromycin, the target of drug inhibition during the first cycle of growth is unknown, and the potential of this novel mechanism of action as a clinical treatment remains unclear.

Several lines of evidence indicate that azithromycin has a secondary mechanism and target(s) of action to inhibit invasion, in addition to binding to the $50 \mathrm{~S}$ apicoplast ribosomal subunit as described for the delayed death' phenotype [31, 32]. Clindamycin, a smaller and structurally unrelated antibiotic that has overlapping binding sites to azithromycin on the apicoplast ribosome [39], as well as an identical mode of action, had very little invasion inhibitory activity. Selection of a D10 line for resistance (57-fold reduced sensitivity) to azithromycin in the 2 cycle 'delayed death' assays did not result in a similar increase in resistance to azithromycin invasion inhibition assays when compared to a parental D10 ('delayed death' sensitive) line. Comparison of azithromycin and its modified analogues $(1 \mathrm{j}, 12 \mathrm{e})$ showed that modifications increasing the potency of these analogues over azithromycin in invasion inhibition, in cycle (40 hour) and 1 cycle (90 hour) assays did not appreciably increase the potency of the compounds over 2 cycle 'delayed death' assays, suggesting the mechanism of enhanced inhibitory activity was independent of anti-ribosomal activity.

The near instantaneous inhibition of merozoite invasion caused by azithromycin also suggests that this drug can rapidly kill parasites through a secondary mechanism of action. Typical 'delayed death' inhibition by macrolide antibiotics results in the loss of apicoplast functionality [31,32], with this then leading to the loss of isoprenoid precursor biosynthesis, the only essential function of the apicoplast during blood-stage development [33]. The long timeframes required for apicoplast translation inhibition and the resulting 'delayed death' caused by macrolide antibiotics is in striking contrast to the very rapid inhibition of merozoite invasion at higher drug concentrations. Furthermore, removal of the apicoplast in the experiments by Yeh et al. [33] was not reported to result in an invasion defect. Combined, the available evidence suggests it is very unlikely that the apicoplast plays some as yet undefined role in merozoite invasion and implicates a secondary mechanism of action of azithromycin against invading merozoites. Whether the mechanism of invasion inhibition and intracellular asexual-stage parasite growth inhibition (within 1 cycle in the absence of invasion inhibition) is the same remains to be elucidated. However, the similar 
$\mathrm{IC}_{50}$ values seen for both inhibitory phenotypes raises the intriguing possibility that this secondary mechanism of action could be targeted throughout the disease causing blood-stage of $P$. falciparum malaria.

The ability of azithromycin to rapidly inhibit merozoite invasion, in addition to the apicoplast-targeting 'delayed death' mechanism of action, is an exciting prospect for the development of macrolide-based drugs in combination therapies. This dual modality could see azithromycin assist in rapid clearance of parasites as well as offering longer-term treatment of the remaining lowlevel blood and liver-stage parasites through its activity against the apicoplast. Such a drug treatment strategy has the potential to increase drug efficacy, while reducing the chances for the development of resistance.

Although the in vitro merozoite invasion inhibitory activity of azithromycin required drug concentrations at the upper end of what is achieved clinically [29, 32], we demonstrate that modification of the macrolides can lead to a substantial improvement in invasion inhibitory activity; this suggests that invasion inhibitory macrolides could be developed as therapeutics. Addition of an L-megosamine sugar to erythromycin A through an in vivo fermentation process [41] produced a compound with 32-fold greater invasion inhibitory activity than parent erythromycin A. Furthermore, a selection of azithromycin analogues [42-44] lowered the invasion inhibitory $\mathrm{IC}_{50}$ by up to 5 -fold. The azithromycin derivatives were tested only when solubilized in DMSO due to limitations in the amount of available material. Importantly, the use of DMSO as vehicle for solubilization reduced the potency of the parent compound azithromycin 4-fold relative to ethanol as vehicle. If this observation were to hold true for the derivatives, the invasion inhibitory $\mathrm{IC}_{50}$ of the compound $1 \mathrm{j}$ (5) [44] would be in the range of $1-2 \mu \mathrm{M}$.

The invasion inhibitory $\mathrm{IC}_{50}$ of azithromycin $(10 \mu \mathrm{M})$ and analogues $(1 \mathrm{j}, 7 \mu \mathrm{M} ; 12 \mathrm{e}, 15 \mu \mathrm{M})$ is comparable to that achieved for inhibitors that specifically target the function of essential merozoite proteins. Two recent proof-of-concept studies identified small molecule invasion inhibitory compounds targeting interactions between essential invasion ligands RON4-AMA1 (inhibitory range 30-6 $\mu \mathrm{M}$ ) and MSP1-19 (replication inhibition $21.7 \mu \mathrm{M})[12,14]$, and reported invasion inhibitory $\mathrm{IC}_{50}$ values in a very similar range to azithromycin and analogues. Another highly selective invasion inhibitor, the 3D7-AMA1-specific peptide inhibitor R1 [52], has an invasion inhibitory $\mathrm{IC}_{50}$ of $2.5 \mu \mathrm{M}$ in assays using purified merozoites [18]. Azithromycin, with minimal modification, can rapidly inhibit merozoite invasion at concentrations comparable to some of the best and most selective invasion inhibitors available for drug development and research. The encouraging improvements evident in the invasion inhibitory activity of azithromycin from only a small panel of derivatives, combined with the proven safety and efficacy of macrolides, support the potential for developing such compounds as therapeutics.

Removal of azithromycin's glycan groups provided insight into the structural requirements for invasion inhibitory activity. Removal of the cladinosyl sugar resulted in a 5-fold reduction in invasion inhibitory activity. Strikingly, removal of both sugars resulted in complete loss of invasion inhibitory activity, strongly suggesting that the desosamine sugar, rather than the cladinosyl, is critical for invasion inhibitory activity. Interestingly, the desosamine sugar is also considered the critical glycosylated group in binding of macrolides to microbial ribosomes [39] with clinically used macrolide antibiotics such as telithromycin having dispensed with the cladinosyl group altogether. Screening of a larger number of macrolides and modified analogues will help identify others with greater potency, and allow assessment of which modifications and groups are most important for activity.

The fact that azithromycin inhibits host invasion by Plasmodium merozoites (from human and rodent malaria) and $T$. gondii tachyzoites parasites suggests that the target of inhibition is shared amongst some apicomplexan invasive stages. The fact that the potency of azithromycin against $P$. falciparum (human) and $P$. berghei (rodent) malarias was very similar raises the possibility that targeting this pathway could be an effective strategy to treat other malaria species. The results of the T. gondii invasion assays suggest that host cell invasion of this more distantly related apicomplexan parasite can also be targeted by azithromycin, albeit at higher concentrations. Importantly, the analogue 12e had equal or better invasion inhibitory potency than azithromycin (in ethanol or DMSO, respectively) when tested at a 6-fold lower concentration. This strongly suggests that analogues of azithromycin could be developed with superior inhibitory activity against tachyzoite invasion and confirms that the invasion inhibitory activity of azithromycin and analogues is shared across distantly related apicomplexan parasites.

Macrolide antibiotics have been linked to mechanisms of action other than binding to microbial ribosomes, including interference with intracellular signalling mechanisms such as $\mathrm{Ca}^{2+}$ and MAPK through an as yet undefined mechanism (reviewed in [53]). Intracellular $\mathrm{Ca}^{2+}$ signalling is thought to play an important role in invasion (reviewed in [54]) so interference with this mechanism by macrolide antibiotics could contribute to inhibition of invasion. Macrolide antibiotics have also been shown to bind to negatively charged phospholipid bilayers and interfere with normal membrane function $[55,56]$. Intriguingly, the relative activity of the 
macrolides appeared to be in part related to the number of cationic groups present on the macrolide.

\section{Conclusions}

The results of this study have identified for the first time that macrolide antibiotics, a diverse group of welltolerated antibiotic drugs with antimalarial properties, inhibit Plasmodium spp. merozoite invasion into erythrocytes in vitro. Invasion inhibition assays and live filming experiments show that azithromycin acts rapidly to stop merozoite entry very early during the invasion process. The mechanism of invasion inhibition appears to be independent of apicoplast ribosomal translation inhibition, which is the currently described mechanism of action of these drugs. Modification of both azithromycin and erythromycin A resulted in promising reductions in the invasion inhibitory $\mathrm{IC}_{50}$ to levels nearing those achieved clinically for the parent compounds, providing a proof-of-concept for the potential development of potent invasion inhibitors. Elucidation of the mechanism of action and subsequent improvements in compound potency could pave the way for development of macrolide antibiotics with dual modality as novel and potent antimalarials to complement frontline treatments for malaria. Furthermore, the use of drugs that inhibit merozoite invasion in combination with drugs that inhibit intra-erythrocytic parasite development may be an effective strategy to facilitate rapid parasite clearance to help improve clinical outcomes, reduce transmission, and minimize the development of resistance.

\section{Methods}

\section{Culture of $P$. falciparum}

D10 parental and GFP fluorescent D10-PfPHG [57] parasites were cultured in human $\mathrm{O}^{+}$erythrocytes (Australian Red Cross, Victoria, Australia) according to established protocols [57, 58]. Synchronization using heparin for growth inhibition and merozoite invasion inhibition assays has been described previously [13, 18, 23, 57]. Unless otherwise stated, the D10-PfPHG isolate was used in assays because the GFP label facilitates accurate quantification of parasite stages by flow cytometry $[13,18]$.

\section{Drug inhibition assays}

A diagram outlining the different drug inhibition assays used in this study to measure $P$. falciparum growth and invasion inhibition is available in Fig. 1a,b,c,d,e. The protocol for measuring drug inhibition of ring- and trophozoite-treated parasites in cycle (approximately 40 hours post-invasion), 1 cycle (90 hours post-invasion, includes 1 cycle of replication) and 2 cycle (120 hour post invasion, includes 2 cycles of replication) growth assays has been described in detail previously $[13,57,59]$. Parasitaemia was measured at trophozoite stage by flow cytometry (BD FACSCalibur, BD Biosciences, Franklin Lakes, NJ, USA) after staining with $10 \mu \mathrm{g} / \mathrm{ml}$ ethidium bromide (EtBr) for 1 hour prior to washing in PBS. Typically, 20,000 to 40,000 erythrocytes were counted for each well. Samples were analyzed using FlowJo software (Tree Star Inc, Ashland, OR, USA).

\section{Invasion inhibition assays}

The protocols for filtration of viable $P$. falciparum and $P$. berghei merozoites from E64-treated schizonts and merozoite invasion inhibition assays have been previously described [13, 18, 45]. Parasitaemia was assessed by flow cytometry of EtBr-treated rings (1 hour postinvasion, $5 \mu \mathrm{g} / \mathrm{ml} \mathrm{EtBr}$, staining 10 minutes, no wash) and trophozoite stages (as per 1 cycle assay). Early ringstage parasites were gated using a Fl-1 high (GFP) and Fl-2 low (EtBr) gate. Trophozoites were gated using a Fl-1 high (GFP) and Fl-2 high (EtBr) gate. Parasitized erythrocytes for microscopy were fixed in methanol and stained for 10 minutes in $10 \%$ Giemsa (Merck, Kenilworth, NJ, USA). For microscopy of fixed slides, the number of early ring-stage parasites in a minimum of 1,000 RBCs was assessed by an experienced microscopist in duplicate wells from three independent experiments.

Liver-stage assays used P. berghei ANKA sporozoites dissected from infected Anopheles stephensi mosquitoes. Two-color invasion assays followed the protocol of Sinnis et al. [60] with the following modification. P. berghei constitutively expressing GFP [61] were added to cultured HepG2 cells in the presence of inhibitory compounds, centrifuged for 3 minutes at $150 \mathrm{~g}$ and then incubated for 1 hour to allow invasion. The liver cell and parasites were then fixed but not permeabilized. When probed with polyclonal antibody against $P$. berghei merozoite surface proteins [62] (500:1) followed by antimouse Alexa Fluor 546 (1,000:1; Invitrogen, Carlsbad, CA, USA) only uninvaded sporozoites are labelled. Without permeabilization, the antibodies cannot pass through the HepG2 cell plasma membrane, so sporozoites within the liver cells are not labelled. Invasion rate is calculated by comparing the number of labelled sporozoites to the total number based on GFP expression. Successful infection of hepatocytes was measured through an extension of the invasion assay by adding the parasites as above, incubating at $37{ }^{\circ} \mathrm{C}$ for 2 hours, exchanging media and then incubating the liver-stage parasites for a further 24 hours to allow development of any intracellular parasites. The presence of developing liver-stage parasites that had successfully invaded and established infection was then quantified according to previously published methods [63].

Toxoplasma gondii invasion assays were performed using the Rh parasite strain according to existing protocols [64] with the following modification. Inhibitory 
compounds were added to both the Endo and invasion buffers prior to commencement of host cell invasion. Parasites were allowed to invade for 15 minutes, fixed and sequentially labeled with $\alpha$ SAG1 (2,000:1) and $\alpha$ GAP45 antibodies (500:1), followed by secondary labeling with Alexa Fluor 488/543 (Invitrogen). Parasites were imaged with a Leica SP2 confocal microscope (Leica, WETZLAR, Germany) and a minimum of 1,000 parasites were counted per well with three replicates for each concentration.

The $\mathrm{IC}_{50}$ and $\mathrm{IC}_{80}$ concentrations were determined using GraphPad Prism (GraphPad Software, La Jolla, CA, USA) as previously described [13]. The inhibitory concentration (IC) for 1 cycle (90 hour), 2 cycle ('delayed death', 120 hour) and merozoite ( $<1$ hour post-invasion) will be discriminated by the addition of $90 \mathrm{hr}, 120 \mathrm{hr}$ and ${ }^{\text {mero }}$ where appropriate.

\section{Live cell microscopy}

Live cell microscopy experiments were undertaken according to the method of Weiss et al. [38]. Briefly, highly synchronous late-stage schizonts were diluted 1:25 in media \pm drug treatment and allowed to settle to produce a monolayer onto a $35 \mathrm{~mm}$ FluoroDish (World Precision Instruments, Sarasota, FL, USA). At $37{ }^{\circ} \mathrm{C}$ on a Zeiss Axio Observer.Z1 fluorescence microscope (Zeiss, Oberkochen, Germany) equipped with humidified gas chamber $\left(90 \% \mathrm{~N}_{2}, 5 \% \mathrm{O}_{2}\right.$ and $\left.5 \% \mathrm{CO}_{2}\right)$, selected schizonts were observed until they ruptured and released their merozoites. Time-lapse videos of the invading merozoites were recorded with a high-resolution AxioCam MRm camera (Zeiss). ImageJ was used to perform image analysis and GraphPad Prism used to perform statistical analyses using a Chi-squared test.

\section{Chemistry}

A summary of the chemical structures of the compounds used in this study is available in Fig. 2. Azithromycin 1, erythromycin 9, erythromycin oxime 11 and dirithromycin 13 were obtained commercially from AkScientific (Union City, CA, USA). Roxithromycin 12, clindamycin 16 and spiramycin 15 were from Sigma Aldrich (St Louis, MO, USA). 6-O-Megosaminyl erythromycin A 14 was a gift from C Goodman, E Rodriguez and $\mathrm{H}$ Gramajo [41]. Compounds $4-8$ were provided by GlaxoSmithKline (Tres Cantos, Spain). Synthesis of these compounds are as previously described: $4(12 \mathrm{e})$ and $5(1 \mathrm{j})$ [44]; 6 (11c) [42]; and 7 (7/47) and 8 (7/25) [43]. AZRdesclad 2 was prepared according to Istuk et al. [65]. ERY-desclad 10 was prepared following methods of LeMahieu et al. [66]. AZR-desglycan 3 was prepared following similar procedures previously outlined in the literature [67, 68]. In general, compounds were solubilized with ethanol as vehicle, with the exception of the initial characterization of azithromycin (1), where both ethanol and DMSO were compared, and compounds 4-8 (DMSO), owing to the limited amount of material available.

\section{Availability of supporting data}

A tabular summary of data used to generate graphs and perform statistical tests presented in this manuscript (Figs. 3b,c; 4; 5e; 6a; 7d; and 8b,e,f) is available in Additional file 3.

\section{Additional files}

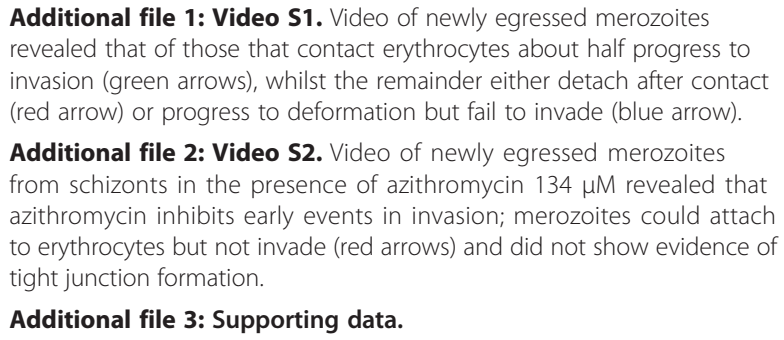

Additional file 2: Video S2. Video of newly egressed merozoites from schizonts in the presence of azithromycin $134 \mu \mathrm{M}$ revealed that azithromycin inhibits early events in invasion; merozoites could attach to erythrocytes but not invade (red arrows) and did not show evidence of tight junction formation.

Additional file 3: Supporting data.

\section{Abbreviations}

DMSO: Dimethyl sulfoxide; EtBr: Ethidium bromide; GFP: Green fluorescent protein; RBC: Red blood cell; SEM: Standard error of the mean; WHO: World Health Organization.

\section{Competing interests}

The authors declare that they have no competing financial interests.

\section{Authors' contributions}

DWW and JGB conceived the study. DWW, CDG, BES, PRG, NWMdJ, FA, CL, JB, BSC, GIM and JGB designed, performed and interpreted experimental data. DWW, CDG, PRG, BES and JGB wrote the paper. All authors read and approved the final manuscript.

\section{Acknowledgements}

We thank Dr Francisco Javier Gamo and Dr José Maria Bueno-Calderón (GlaxoSmithKline, Tres Cantos facility, Spain) for the provision of modified azalides. Prof. Alan Cowman, The Walter \& Eliza Hall Institute, for advice and support throughout the study. Human erythrocytes were kindly provided by the Red Cross Blood Bank (Melbourne, Australia). This work was made possible through Victorian State Government Operational Infrastructure Support and Australian Government National Health and Medical Research Council (NHMRC) Independent Medical Research Institutes Infrastructure Support Scheme (IRIISS). Funding for the research came from the NHMRC of Australia (program grant 637406 to BSC, GIM and JGB) and a Career Development Award to JGB; Peter Doherty Australian Biomedical Fellowship to DWW (APP1035715); postgraduate research scholarship to FA (APP1055246), the Australian Research Council (Future Fellowship to JGB (FT0992317); JB (FT100100112)) and the Australian Cancer Research Foundation. JB is currently supported by a new Investigator Award from the Wellcome Trust (100993/Z/13/ Z). GIM is a Howard Hughes International Scholar. The funders had no role in study design, data collection and analysis, decision to publish, or preparation of the manuscript. The authors have declared that no conflict of interest exists.

\section{Author details}

${ }^{1}$ Research Centre for Infectious Diseases, School of Biological Sciences, The University of Adelaide, Adelaide, South Australia. ${ }^{2}$ Walter and Eliza Hall Institute of Medical Research, Parkville, Victoria 3050, Australia. ${ }^{3}$ Department of Medical Biology, University of Melbourne, Parkville, Victoria 3050, Australia. ${ }^{4}$ Macfarlane Burnet Institute for Medical Research and Public Health, Melbourne, Victoria 3004, Australia. ${ }^{5}$ Plant Cell Biology Research Centre, School of Biosciences, University of Melbourne, Parkville, Victoria 3010, Australia. 'Department of Life Sciences, Imperial College London, South Kensington, London SW7 2AZ, UK. `Department of Immunology, Monash 
University, Clayton, Victoria 3800, Australia. ${ }^{8}$ Department of Microbiology, Monash University, Clayton, Victoria 3168, Australia.

Received: 26 January 2015 Accepted: 30 June 2015 Published online: 18 July 2015

\section{References}

1. World Health Organization (WHO). World malaria report 2014. Geneva: WHO; 2014

2. Ferone R, Burchall JJ, Hitchings GH. Plasmodium berghei dihydrofolate reductase. Isolation, properties, and inhibition by antifolates. Mol Pharmacol. 1969:5:49-59.

3. Zarchin S, Krugliak M, Hinsburg H. Digestion of the host erythrocyte by malaria parasites is the primary target for quinoline containing antimalarials. Biochem Pharmacol. 1986;35:2435-42.

4. Loria P, Miller S, Foley M, Tilley L. Inhibition of the peroxidative degradation of haem as the basis of action of chloroquine and other quinoline antimalarials. Biochem J. 1999;339:363-70.

5. Krishna S, Uhlemann AC, Haynes RK. Artemisinins: mechanisms of action and potential for resistance. Drug Resist Updat. 2004;7:233-44.

6. Famin $\mathrm{O}$, Ginsburg $\mathrm{H}$. Differential effects of 4-aminoquinoline-containing antimalarial drugs on hemoglobin digestion in Plasmodium falciparuminfected erythrocytes. Biochem Pharmacol. 2002;63:393-8.

7. Noedl H, Socheat D, Satimai W. Artemisinin-resistant malaria in Asia. N Engl J Med. 2009;361:540-1.

8. Dondorp AM, Nosten F, Yi P, Das D, Phyo AP, Tarning J, et al. Artemisinin resistance in Plasmodium falciparum malaria. N Engl J Med. 2009;361:455-67.

9. Ashley EA, Dhorda M, Fairhurst RM, Amaratunga C, Lim P, Suon S, et al. Spread of artemisinin resistance in Plasmodium falciparum malaria. $N$ Engl J Med. 2014;371:411-23.

10. World Health Organization (WHO). Emergency response to artemisinin resistance in the Greater Mekong subregion: regional framework for action 2013-2015. Geneva: WHO; 2013.

11. Boyle MJ, Wilson DW, Beeson JG. New approaches to studying Plasmodium falciparum merozoite invasion and insights into invasion biology. Int J Parasitol. 2013;43:1-10.

12. Srinivasan P, Yasgar A, Luci DK, Beatty WL, Hu X, Andersen J, et al. Disrupting malaria parasite AMA1-RON2 interaction with a small molecule prevents erythrocyte invasion. Nat Commun. 2013;4:2261.

13. Wilson DW, Langer C, Goodman CD, McFadden GI, Beeson JG. Defining the timing of action of antimalarial drugs against Plasmodium falciparum. Antimicrob Agents Chemother. 2013;57:1455-67.

14. Chandramohanadas R, Basappa, Russell B, Liew K, Yau YH, Chong A, et al. Small molecule targeting malaria merozoite surface protein-1 (MSP-1) prevents host invasion of divergent plasmodial species. J Infect Dis. 2014;210:1616-26.

15. Teuscher F, Gatton ML, Chen N, Peters J, Kyle DE, Cheng Q. Artemisinininduced dormancy in plasmodium falciparum: duration, recovery rates, and implications in treatment failure. J Infect Dis. 2010;202:1362-8.

16. Hughes KR, Biagini GA, Craig AG. Continued cytoadherence of Plasmodium falciparum infected red blood cells after antimalarial treatment. Mol Biochem Parasitol. 2010;169:71-8.

17. Barnes Kl, Little F, Mabuza A, Mngomezulu N, Govere J, Durrheim D, et al. Increased gametocytemia after treatment: an early parasitological indicator of emerging sulfadoxine-pyrimethamine resistance in falciparum malaria. J Infect Dis. 2008:197:1605-13.

18. Boyle MJ, Wilson DW, Richards JS, Riglar DT, Tetteh KK, Conway DJ, et al. Isolation of viable Plasmodium falciparum merozoites to define erythrocyte invasion events and advance vaccine and drug development. Proc Natl Acad Sci U S A. 2010;107:14378-83.

19. Riglar DT, Richard D, Wilson DW, Boyle MJ, Dekiwadia C, Turnbull L, et al. Super-resolution dissection of coordinated events during malaria parasite invasion of the human erythrocyte. Cell Host Microbe. 2011;9:9-20.

20. Marapana DS, Wilson DW, Zuccala ES, Dekiwadia CD, Beeson JG, Ralph SA et al. Malaria parasite signal peptide peptidase is an ER-resident protease required for growth but not for invasion. Traffic. 2012;13:1457-65.

21. Dejkriengkraikhul $P$, Wilairat $P$. Requirement of malarial protease in the invasion of human red cells by merozoites of Plasmodium falciparum. Z Parasitenkd. 1983;69:313-7.
22. Dluzewski AR, Rangachari K, Wilson RJ, Gratzer WB. Plasmodium falciparum: protease inhibitors and inhibition of erythrocyte invasion. Exp Parasitol. 1986;62:416-22.

23. Boyle MJ, Richards JS, Gilson PR, Chai W, Beeson JG. Interactions with heparin-like molecules during erythrocyte invasion by Plasmodium falciparum merozoites. Blood. 2010;115:4559-68.

24. Vaid A, Thomas DC, Sharma P. Role of Ca2+/calmodulin-PfPKB signaling pathway in erythrocyte invasion by Plasmodium falciparum. J Biol Chem. 2008;283:5589-97.

25. Sykes A, Hendriksen I, Mtove G, Mandea V, Mrema H, Rutta B, et al. Azithromycin plus artesunate versus artemether-lumefantrine for treatment of uncomplicated malaria in Tanzanian children: a randomized, controlled trial. Clin Infect Dis. 2009;49:1195-201.

26. Ramharter M, Oyakhirome $S$, Klein Klouwenberg P, Adegnika AA, Agnandji ST, Missinou MA, et al. Artesunate-clindamycin versus quinine-clindamycin in the treatment of Plasmodium falciparum malaria: a randomized controlled trial. Clin Infect Dis. 2005;40:1777-84.

27. Noedl H, Krudsood S, Chalermratana K, Silachamroon U, Leowattana W, Tangpukdee $\mathrm{N}$, et al. Azithromycin combination therapy with artesunate or quinine for the treatment of uncomplicated Plasmodium falciparum malaria in adults: a randomized, phase 2 clinical trial in Thailand. Clin Infect Dis. 2006:43:1264-71

28. Nosten F, McGready R, d'Alessandro U, Bonell A, Verhoeff F, Menendez C, et al. Antimalarial drugs in pregnancy: a review. Curr Drug Saf. 2006;1:1-15.

29. Cook JA, Randinitis EJ, Bramson CR, Wesche DL. Lack of a pharmacokinetic interaction between azithromycin and chloroquine. Am J Trop Med Hyg. 2006:74:407-12.

30. Fichera ME, Roos DS. A plastid organelle as a drug target in apicomplexan parasites. Nature. 1997:390:407-9.

31. Goodman CD, Su V, McFadden Gl. The effects of anti-bacterials on the malaria parasite Plasmodium falciparum. Mol Biochem Parasitol. 2007;152:181-91.

32. Dahl EL, Rosenthal PJ. Multiple antibiotics exert delayed effects against the Plasmodium falciparum apicoplast. Antimicrob Agents Chemother. 2007;51:3485-90

33. Yeh E, DeRisi JL. Chemical rescue of malaria parasites lacking an apicoplast defines organelle function in blood-stage Plasmodium falciparum. PLoS Biol. 2011;9, e1001138.

34. van Eijk AM, Terlouw DJ. Azithromycin for treating uncomplicated malaria. Cochrane Database Syst Rev. 2011;2, CD006688.

35. Gilson PR, Crabb BS. Morphology and kinetics of the three distinct phases of red blood cell invasion by Plasmodium falciparum merozoites. Int J Parasitol. 2009:39:91-6.

36. Miller LH, Aikawa M, Dvorak JA. Malaria (Plasmodium knowlesi) merozoites: immunity and the surface coat. J Immunol. 1975;114:1237-42.

37. Yahata K, Treeck M, Culleton R, Gilberger TW, Kaneko O. Time-lapse imaging of red blood cell invasion by the rodent malaria parasite Plasmodium yoelii. PLoS One. 2012;7, e50780.

38. Weiss GE, Gilson PR, Taechalertpaisarn T, Tham WH, de Jong NW, Harvey KL, et al. Revealing the sequence and resulting cellular morphology of receptor-ligand interactions during Plasmodium falciparum invasion of erythrocytes. PLoS Pathog. 2015;11, e1004670.

39. Schlunzen F, Zarivach R, Harms J, Bashan A, Tocilj A, Albrecht R, et al. Structural basis for the interaction of antibiotics with the peptidyl transferase centre in eubacteria. Nature. 2001;413:814-21.

40. Bowers AK, Keller JA, Dutcher SK. Molecular markers for rapidly identifying candidate genes in Chlamydomonas reinhardtii. Ery 1 and ery2 encode chloroplast ribosomal proteins. Genetics. 2003;164:1345-53.

41. Goodman CD, Useglio M, Peiru S, Labadie GR, McFadden GI, Rodriguez E, et al. Chemobiosynthesis of new antimalarial macrolides. Antimicrob Agents Chemother. 2013;57:907-13.

42. Peric M, Fajdetic A, Rupcic R, Alihodzic S, Ziher D, Bukvic Krajacic M, et al. Antimalarial activity of 9a-N substituted 15-membered azalides with improved in vitro and in vivo activity over azithromycin. J Med Chem. 2012;55:1389-401.

43. Hutinec A, Rupcic R, Ziher D, Smith KS, Milhous W, Ellis W, et al. An automated, polymer-assisted strategy for the preparation of urea and thiourea derivatives of 15-membered azalides as potential antimalarial chemotherapeutics. Bioorg Med Chem. 2011;19:1692-701.

44. Bukvic Krajacic M, Peric M, Smith KS, Schonfeld ZI, Ziher D, Fajdetic A, et al. Synthesis, structure-activity relationship, and antimalarial activity of ureas and thioureas of 15-membered azalides. J Med Chem. 2011;54:3595-605. 
45. Angrisano F, Riglar DT, Sturm A, Volz JC, Delves MJ, Zuccala ES, et al. Spatial localisation of actin filaments across developmental stages of the malaria parasite. PLoS One. 2012;7, e32188.

46. Friesen J, Silvie O, Putrianti ED, Hafalla JC, Matuschewski K, Borrmann S. Natural immunization against malaria: causal prophylaxis with antibiotics. Sci Transl Med. 2010;2:40ra49.

47. Baum J, Gilberger TW, Frischknecht F, Meissner M. Host-cell invasion by malaria parasites: insights from Plasmodium and Toxoplasma. Trends Parasitol. 2008;24:557-63.

48. Teo CF, Zhou XW, Bogyo M, Carruthers VB. Cysteine protease inhibitors block Toxoplasma gondii microneme secretion and cell invasion. Antimicrob Agents Chemother. 2007;51:679-88.

49. Gingras BA, Jensen JB. Activity of azithromycin (CP-62,993) and erythromycin against chloroquine-sensitive and chloroquine-resistant strains of Plasmodium falciparum in vitro. Am J Trop Med Hyg. 1992;47:378-82.

50. Sidhu AB, Sun Q, Nkrumah $\sqcup$, Dunne MW, Sacchettini JC, Fidock DA. In vitro efficacy, resistance selection, and structural modeling studies implicate the malarial parasite apicoplast as the target of azithromycin. J Biol Chem. 2007;282:2494-504.

51. Unger HW, Ome-Kaius M, Wangnapi RA, Umbers AJ, Hanieh S, Suen CS, et al. Sulphadoxine-pyrimethamine plus azithromycin for the prevention of low birthweight in Papua New Guinea: a randomised controlled trial. BMC Med. 2015;13:9.

52. Harris KS, Casey JL, Coley AM, Masciantonio R, Sabo JK, Keizer DW, et al. Binding hot spot for invasion inhibitory molecules on Plasmodium falciparum apical membrane antigen 1. Infect Immun. 2005;73:6981-9.

53. Kanoh S, Rubin BK. Mechanisms of action and clinical application of macrolides as immunomodulatory medications. Clin Microbiol Rev. 2010;23:590-615.

54. Lim DC, Cooke BM, Doerig C, Saeij JP. Toxoplasma and Plasmodium protein kinases: roles in invasion and host cell remodelling. Int J Parasitol. 2012;42:21-32.

55. Montenez JP, Van Bambeke F, Piret J, Brasseur R, Tulkens PM, Mingeot-Leclercq MP. Interactions of macrolide antibiotics (Erythromycin A, roxithromycin, erythromycylamine [Dirithromycin], and azithromycin) with phospholipids: computer-aided conformational analysis and studies on acellular and cell culture models. Toxicol Appl Pharmacol. 1999;156:129-40.

56. Tyteca D, Schanck A, Dufrene YF, Deleu M, Courtoy PJ, Tulkens PM, et al. The macrolide antibiotic azithromycin interacts with lipids and affects membrane organization and fluidity: studies on Langmuir-Blodgett monolayers, liposomes and $\mathbf{J 7 7 4}$ macrophages. J Membr Biol. 2003;192:203-15.

57. Wilson DW, Crabb BS, Beeson JG. Development of fluorescent Plasmodium falciparum for in vitro growth inhibition assays. Malar J. 2010;9:152.

58. Trager W, Jensen JB. Human malaria parasites in continuous culture. Science. 1976;193:673-5.

59. Persson KE, Lee CT, Marsh K, Beeson JG. Development and optimization of high-throughput methods to measure Plasmodium falciparum-specific growth inhibitory antibodies. J Clin Microbiol. 2006;44:1665-73.

60. Sinnis P, De La Vega P, Coppi A, Krzych U, Mota MM. Quantification of sporozoite invasion, migration, and development by microscopy and flow cytometry. Methods Mol Biol. 2013;923:385-400.

61. Franke-Fayard B, Trueman H, Ramesar J, Mendoza J, van der Keur M, van der Linden $\mathrm{R}$, et al. A Plasmodium berghei reference line that constitutively expresses GFP at a high level throughout the complete life cycle. Mol Biochem Parasitol. 2004;137:23-33.

62. Nair SC, Brooks CF, Goodman CD, Sturm A, McFadden GI, Sundriyal S, et al. Apicoplast isoprenoid precursor synthesis and the molecular basis of fosmidomycin resistance in Toxoplasma gondii. J Exp Med. 2011;208:1547-59.

63. Sumanadasa SD, Goodman CD, Lucke AJ, Skinner-Adams T, Sahama I, Haque A, et al. Antimalarial activity of the anticancer histone deacetylase inhibitor SB939. Antimicrob Agents Chemother. 2012:56:3849-56.

64. Alexander DL, Mital J, Ward GE, Bradley P, Boothroyd JC. Identification of the moving junction complex of Toxoplasma gondii: a collaboration between distinct secretory organelles. PLoS Pathog. 2005;1, e17.

65. Istuk ZM, Mutak S, Kujundzic N, Kragol G. Novel 9a-carbamoyl- and 9a-thiocarbamoyl-3-decladinosyl-6-hydroxy and 6-methoxy derivatives of 15-membered macrolides. Bioorg Med Chem. 2007;15:4498-510.
66. LeMahieu RA, Carson M, Kierstead RW, Fern LM, Grunberg E. Glycoside cleavage reactions on erythromycin A. Preparation of erythronolide A. J Med Chem. 1974;17:953-6.

67. Mercep M, Mesic M, Tomaskovic L, Markovic S, Poljak V, Sijan G, Selmani S Conjugates with anti-inflammatory activity. World Intelectual Property Organization. WO 2006/046123 A2;2006.

68. Djokic S, Kobrehel G, Lopotar N, Kamenar B, Nagl D, Mrvos D. Erythromycin series. Part 13. Synthesis and structure elucidation of 10-dihydro-10deoxo-methyl-11-azaerythromycin A. J Chem Res (S). 1988:152-153.

\section{Submit your next manuscript to BioMed Central and take full advantage of:}

- Convenient online submission

- Thorough peer review

- No space constraints or color figure charges

- Immediate publication on acceptance

- Inclusion in PubMed, CAS, Scopus and Google Scholar

- Research which is freely available for redistribution

Submit your manuscript at www.biomedcentral.com/submit 